INTERNATIONAL JOURNAL FOR NUMERICAL METHODS IN ENGINEERING

Int. J. Numer. Meth. Engng 2010; 00:1-6 Prepared using nmeauth.cls [Version: 2002/09/18 v2.02]

\title{
Velocity-based formulations for standard and quasi-incompressible hypoelastic-plastic solids
}

\author{
Alessandro Franci ${ }^{1}$, Eugenio Oñate ${ }^{1 *}$, Josep Maria Carbonell ${ }^{2}$ \\ 1 Centre Internacional de Mètodes Numèrics en Enginyeria (CIMNE) \\ Campus Norte UPC, 08034 Barcelona, Spain \\ Email: falessandro,onate@cimne.upc.edu \\ 2 Universitat Politècnica de Catalunya (UPC), Email: cpuigbo@cimne.upc.edu
}

\begin{abstract}
SUMMARY
We present three velocity-based Updated Lagrangian formulations for standard and quasiincompressible hypoelastic-plastic solids. Three finite elements, named V, VP and VPS elements are derived and tested for benchmark for non-linear solid mechanics problems. The V-element is based on a standard velocity approach, while for the VP and VPS elements a mixed velocity-pressure formulation is used. The two-field problem is solved via a two-step Gauss-Seidel partitioned iterative scheme. First the momentum equations are solved in terms of velocity increments, as for the V-element. Then the constitutive relation for the pressure is solved using the updated velocities obtained at the previous step. For the VPS-element the equation for the pressure is stabilized using the Finite Calculus (FIC) method in order to solve problems involving quasi-incompressible materials. All the solid elements are validated by solving 2D and 3D benchmark problems in statics as in dynamics. Copyright (C) 2010 John Wiley \& Sons, Ltd.
\end{abstract}

KEY WORDS: Finite Element Methods, Lagrangian, Non-linear dynamics, hypoelastic solids.

\section{Introduction}

The objective of this work is the derivation and analysis of three velocity-based Updated Lagrangian (UL) formulations for non-linear solid mechanics. In particular, a purely velocity scheme and two mixed velocity-pressure formulations, with and without stabilization, are presented. From the adaptation of these schemes to the hypoelastic-plastic model, three finite elements are derived, namely, the Velocity (V), the Velocity-Pressure (VP) and the VelocityPressure Stabilized (VPS) elements.

In solid mechanics, velocity-based schemes are not largely used and generally displacementbased approaches are preferred [24]. The main reason is that velocity formulations require a time integration procedure also for computing the stresses of an elastic material, as for the inelastic ones. However, there are some applications in which a velocity formulation may be

${ }^{*}$ Correspondence to: CIMNE, Edificio C1, Campus Norte, UPC, Gran Capitán s/n, 08034 Barcelona, Spain 
preferred. For example, a velocity approach can be useful for the description of solids that undergo huge deformations. In industrial processes, as the extrusion and forming of solids, the plastic (or viscoplastic) deformations are so large that the elastic strain is negligible. In these situations, the problem becomes one of incompressible viscous, non-Newtonian flow and a velocity-based formulation is the most appropriate scheme for its description. This is the main idea of the so-called flow approach $[15,34,35]$. Also in the context of fluid-structure interaction (FSI) problems, the use of a velocity formulation for solids may be very helpful. In fact, this scheme facilitates the coupling with fluids, that are typically solved for the velocities, and allows the solution of FSI problems with a monolithic scheme ensuring in this way a strong FSI coupling $[22,23,2]$. In this sense, the velocity-based formulations derived in this paper represent a further step towards the unified solution of continuum mechanics problems, in which solids and fluids can be computed using the same solver $[17,13]$.

It is well known that one-field formulations, displacement-based or velocity-based indifferently, are not enough for dealing with incompressible materials [1]. In order to avoid numerical drawbacks, as volumetric locking or spurious hydrostatic pressure fluctuations, multi-field elements are required $[9,14,6]$. In this work a mixed Velocity-Pressure scheme is used for both VP and VPS elements.

One of the novelties of this work is the proposal of a new stabilization procedure for the VP formulation and lower order elements. For this reason, linear shape functions have been used for interpolating both nodal velocities and pressures within the elements (triangles in 2D and tetrahedra in 3D). For incompressible or nearly incompressible materials, this combination does not fulfill the inf - sup condition [8] and the numerical scheme needs to be stabilized. The required stabilization is given using a Finite Calculus (FIC) procedure analogous to the one derived in [21]. In the mentioned work the stabilization was derived for quasi-incompressible Newtonian fluids. Here, a similar stabilization procedure, obtained by introducing only small modifications into the scheme proposed in [21], is applied to quasi-incompressible hypoelastic solids and implemented in the VPS-element. This operation is facilitated by the similarity between the constitutive models for hypoelastic solids and quasi-incompressible Newtonian fluids $[17,13]$.

Thanks to its rate-based definition, hypoelasticity could be included in the proposed velocitybased schemes in a natural way. However, note that these formulations do not preclude the extension to other constitutive laws, as hyperelastic models [4]. Using the definition of Truesdell [32], a hypoelastic body is a material which may soften or harden in strain but in general has neither a preferred state nor a preferred stress. The hypoelastic laws were created with the purpose of transfering the linear theory of elasticity from the small to the finite strains regime [32]. In [33] a deep dissertation about the differences between elasticity and hypoelasticity is given. Hypoelastic models are not free of drawbacks [28, 19, 18]. First of all, the Cauchy stresses need to be computed through a time integration and this must be such that the frame invariance of the formulation is preserved. Furthermore, contrary to hyperelastic models, for large deformations the hypoelastic laws do not guarantee that the work done in a closed deformation path is zero [24]. However this error can be considered negligible if the elastic deformations are small versus the total deformations [4]. For this reason, the hypoelastic laws are often used for describing the elastic part of elastic-plastic materials, where the plastic deformations represent the largest part of the overall deformation. This explains why hypoelasto-plastic models have been largely used for modeling forming processes $[7,16,11]$. One of the objectives of this work is to show the reliability of the hypoelastic model despite 
the mentioned inconveniences. This is done by testing the formulation with highly non-linear solid mechanics problems, involving plasticity, large displacements and quasi-incompressible materials. The solutions given by the V, VP and VPS elements are discussed and compared to each other and to other numerical scheme presented in the literature.

This paper is structured as follows. First the hypoelastic-plastic model used in this work is presented. Next the governing equations are given. In section 4 the linearization procedure of linear momentum equations is explained in detail. Then the solution schemes for the $\mathrm{V}$ and the VP elements are given. Next the FIC stabilization is introduced and the solution algorithm for the VPS-element is described. Section 9 is devoted to the validation of the V, VP and VPS elements through the solution of three benchmark problems. Finally the conclusions of this work are given.

\section{Hypoelastic model}

The hypoelastic model is defined by a direct relation between the Cauchy stress rate tensor $\boldsymbol{\sigma} \nabla$ and the deformation rate tensor $\boldsymbol{d}$. Using the Jaumann measure of stress, $\boldsymbol{\sigma} \nabla$ is computed as $[4]$

$$
\sigma^{\nabla}=c^{\nabla J}: d
$$

where the fourth-order tensor $\boldsymbol{c}^{\nabla J}$ is the Jaumann tangent moduli and $\boldsymbol{d}$ is defined as

$$
d_{i j}=\frac{1}{2}\left(\frac{\partial v_{i}}{\partial x_{j}}+\frac{\partial v_{j}}{\partial x_{i}}\right)
$$

For an isotropic body $\boldsymbol{c}^{\nabla J}$ is computed as

$$
c_{i j k l}^{\nabla J}=\kappa \delta_{i j} \delta_{k l}+\mu\left(\delta_{i k} \delta_{j l}+\delta_{i l} \delta_{k j}-\frac{2}{3} \delta_{i j} \delta_{k l}\right) \quad, \quad \boldsymbol{c}^{\nabla J}=\kappa \mathbf{1} \otimes \mathbf{1}+2 \mu \mathbf{I}^{\prime}
$$

where the bulk modulus $\kappa$ is computed from the Lamé parameters, $\lambda$ and $\mu$, as

$$
\kappa=\lambda+\frac{2}{3} \mu,
$$

$\mathbf{1}$ is the second-order identity tensor and $\mathbf{I}^{\prime}$ is a fourth-order tensor computed as

$$
\mathbf{I}^{\prime}=\mathbf{I}-\frac{1}{3} \mathbf{1} \otimes \mathbf{1}
$$

where $\mathbf{I}$ is the fourth-order symmetric identity tensor and it is computed as $\mathrm{I}_{i j k l}=$ $\frac{1}{2}\left(\delta_{i k} \delta_{j l}+\delta_{i l} \delta_{k j}\right)$.

For the computation of the Cauchy stress it is required to integrate on time its material time derivative. This is computed as

$$
\dot{\sigma}=\sigma^{\nabla}+\boldsymbol{\Omega}(\boldsymbol{\sigma})
$$

where $\boldsymbol{\Omega}$ is a tensor that accounts for the rotations and it is required for guaranteing the frame invariance of the scheme. This tensor is defined as

$$
\boldsymbol{\Omega}=\boldsymbol{W} \cdot \boldsymbol{\sigma}+\boldsymbol{\sigma} \cdot \boldsymbol{W}^{T}
$$


where $\boldsymbol{W}$ is the spin tensor defined as

$$
W_{i j}=\frac{1}{2}\left(\frac{\partial v_{i}}{\partial x_{j}}-\frac{\partial v_{j}}{\partial x_{i}}\right)
$$

Discretizing in time Eq.(6) for the time step interval $\left[{ }^{n} t,{ }^{n+1} t\right]$ and replacing $\boldsymbol{\sigma}$ with Eq.(1), yields

$$
\frac{{ }^{n+1} \boldsymbol{\sigma}-{ }^{n} \boldsymbol{\sigma}}{\Delta t}=\boldsymbol{c}^{\nabla J}:{ }^{n+1} \boldsymbol{d}+\boldsymbol{\Omega}\left({ }^{n} \boldsymbol{\sigma}\right)
$$

$\boldsymbol{\Omega}$ is viewed as a correction of the Cauchy stress tensor. Thus the two tensors are joined as

$$
{ }^{n} \hat{\boldsymbol{\sigma}}={ }^{n} \boldsymbol{\sigma}+\Delta t \boldsymbol{\Omega}\left({ }^{n} \boldsymbol{\sigma}\right)
$$

Note that the explicit computation of $\boldsymbol{\Omega}$ is allowed only if the solid undergoes small rotations within a time step increment. Otherwise, a proper objective integration algorithm should be used [4].

Substituting Eq.(10) in Eq.(9), yields

$$
\frac{{ }^{n+1} \boldsymbol{\sigma}-{ }^{n} \hat{\boldsymbol{\sigma}}}{\Delta t}=\boldsymbol{c}^{\nabla J}:{ }^{n+1} \boldsymbol{d}
$$

Substituting in Eq.(11) the relation for $\boldsymbol{c}^{\nabla J}$ using Eq.(3), yields

$$
\frac{{ }^{n+1} \boldsymbol{\sigma}-{ }^{n} \hat{\boldsymbol{\sigma}}}{\Delta t}=\left(\kappa \mathbf{1} \otimes \mathbf{1}+2 \mu \mathbf{I}^{\prime}\right):{ }^{n+1} \boldsymbol{d}
$$

Hence,

$$
{ }^{n+1} \boldsymbol{\sigma}={ }^{n} \hat{\boldsymbol{\sigma}}+\kappa \Delta t(\mathbf{1} \otimes \mathbf{1}):{ }^{n+1} \boldsymbol{d}+2 \mu \Delta t \mathbf{I}^{\prime}:{ }^{n+1} \boldsymbol{d}
$$

The second term of the right hand side of Eq.(13) represents the increment of the volumetric, or pressure, part of the Cauchy stress. Thus, the following relation for the increment of pressure holds

$$
\Delta p={ }^{n+1} p-{ }^{n} p=\kappa \Delta t \mathbf{1}:{ }^{n+1} \boldsymbol{d}
$$

Hence,

$$
{ }^{n+1} p={ }^{n} p+\kappa \Delta t^{n+1} d^{v}
$$

where $d^{v}=\mathbf{1}: \boldsymbol{d}$ is the volumetric strain rate.

From Eq.(13) one may compute the updated stresses using the velocities only, or both the pressure and the velocities, as follows

$$
\begin{gathered}
{ }^{n+1} \boldsymbol{\sigma}={ }^{n} \hat{\boldsymbol{\sigma}}+\Delta t\left(\kappa \mathbf{1} \otimes \mathbf{1}+2 \mu \mathbf{I}^{\prime}\right):{ }^{n+1} \boldsymbol{d} \\
{ }^{n+1} \boldsymbol{\sigma}={ }^{n} \hat{\boldsymbol{\sigma}}+\Delta p \mathbf{1}+2 \Delta t \mu \mathbf{I}^{\prime}:{ }^{n+1} \boldsymbol{d}
\end{gathered}
$$

The plastic behavior of the hypoelastic-plastic model is described considering the $J_{2}$ von Mises flow model and an associative plasticity [4]. The von Mises law is particularly indicated for metal plasticity and the associative plastic flow gives important benefits for the symmetry of the tangent matrix [4].

For this model the elastoplastic tangent moduli is [4]

$$
\boldsymbol{c}_{e p}^{\nabla J}=\kappa \mathbf{1} \otimes \mathbf{1}+2 \mu \mathbf{I}^{\prime}-2 \mu \gamma \boldsymbol{n} \otimes \boldsymbol{n}
$$

Int. J. Numer. Meth. Engng 2010; 00:1-6 
with $\gamma=\frac{1}{1+(H / 3 \mu)}$ and $\boldsymbol{n}=\sqrt{\frac{3}{2}} \frac{\boldsymbol{\sigma}^{\prime}}{\bar{\sigma}}$, where $H$ is the plastic modulus and $\bar{\sigma}$ is the effective stress.

Note that the continuum elasto-plastic tangent modulus tensor of this model preserves the symmetry properties of its elastic counterpart. For elastic loading or unloading, $\boldsymbol{c}_{e p}^{\nabla J}=\boldsymbol{c}^{\nabla J}$.

In order to guarantee the consistency of the elastoplastic incremental scheme, a return mapping algorithm is used. The return mapping algorithm consists of an initial trial elastic step followed by a plastic corrector one that is activated when the yield function at a trial elastic step takes a positive value [4]. For the $J_{2}$ flow theory and associative plasticity the return mapping is characterized to be radial with respect to the yield surface [30].

\section{Governing equations}

The motion and deformation of a body are described by the linear momentum equations. These are solved using an Updated Lagrangian (UL) description. In this framework the governing equations are integrated over the unknown configuration $\Omega$ (the so-called updated configuration) and the space derivatives are computed with respect to the spatial coordinates. Thus, the local form of the linear momentum equations reads

$$
\rho \frac{\partial \boldsymbol{v}}{\partial t}-\operatorname{div}(\boldsymbol{\sigma})-\boldsymbol{b}=0 \quad \text { in } \Omega \times(0, T)
$$

where $\rho$ is the density, $\boldsymbol{v}$ is the velocity vector, $\boldsymbol{\sigma}$ is the Cauchy stress tensor and $\boldsymbol{b}$ is the body force vector and $t$ is the time.

The set of governing equations is completed by the following conditions at the Dirichlet $\left(\Gamma_{v}\right)$ and Neumann $\left(\Gamma_{t}\right)$ boundaries

$$
\begin{array}{cc}
v_{i}-v_{i}^{p}=0 & \text { on } \Gamma_{v} \\
\sigma_{i j} n_{j}-t_{i}^{p}=0 & \text { on } \Gamma_{t}
\end{array}
$$

where $v_{i}^{p}$ and $t_{i}^{p}, i=1, \ldots, n$ are the prescribed velocities and the prescribed tractions, respectively.

In the following, summation of terms for repeated indices is assumed unless otherwise specified.

For mixed velocity-pressure elements, an additional equation for the pressure is required. Thus, for VP and VPS elements also the constitutive relation between the volumetric deformation rate and the pressure variation (Eq.(15)) is solved.

\section{Linearization of the momentum equations}

Integrating Eq.(19) over $\Omega$ and after standard transformations, the Galerkin expression of the Principle of Virtual Power [4] in the UL framework for the node $I$ is obtained as

$$
\underbrace{\int_{\Omega} N_{I} \rho d \Omega \dot{v}_{i}}_{f_{I i}^{d y n}}+\underbrace{\int_{\Omega} \frac{\partial N_{I}}{\partial x_{j}} \sigma_{j i} d \Omega}_{f_{I i}^{i n t}}=\underbrace{\int_{\Omega} N_{I} b_{i} d \Omega+\int_{\Gamma_{t}} N_{I} t_{i}^{p} d \Gamma}_{f_{I i}^{\text {ext }}}
$$


where $N$ are the shape functions, $\boldsymbol{x}$ are the spatial coordinates and $\boldsymbol{f}^{d y n}, \boldsymbol{f}^{i n t}$ and $\boldsymbol{f}^{\text {ext }}$ are the dynamic, internal and external forces, respectively, expressed in the UL framework. In this work we use linear shape functions for approximating the velocity and pressure fields.

For convenience, the linearization of the internal forces $f^{i n t}$ is performed in the known configuration $\Omega_{0}$, as for a total Lagrangian (TL) description and then the UL linearized form is obtained via a push-forward transformation of the TL form.

Applying standard pull back tranformations to vector $f^{\text {int }}$ of Eq.(22), the internal forces for a TL description ${ }^{T L} f^{\text {int }}$ read

$$
{ }^{T L} f_{I i}^{i n t}=\int_{\Omega_{0}} \frac{\partial N_{I}}{\partial X_{j}} P_{j i} d \Omega_{0}
$$

where $\boldsymbol{P}$ is the first Piola-Kirchhoff stress tensor (also called nominal stress tensor) and $\boldsymbol{X}$ are the material coordinates. All the variables with subscript $(\cdot)_{0}$ refer to the last known configuration. For the sake of clarity in the notation, the terms referred to the TL description are denoted with the left upper index ${ }^{T L}(\cdot)$. Unless otherwise specified, the variables belong to the UL description.

The hypoelastic constitutive relations are defined for the rates of stresses and strains. Hence it is more convenient to perform the linearization of the material derivative of the internal forces and then integrate for the time step increment $\Delta t$. The material time derivative of Eq. (23) is

$$
{ }^{T L} \dot{f}_{I i}^{i n t}=\int_{\Omega_{0}} \frac{\partial N_{I}}{\partial X_{j}} \dot{P}_{j i} d \Omega_{0}
$$

As the first Piola-Kirchhoff stress tensor $\boldsymbol{P}$ is not symmetric and its rate is a non-objective measure, it is preferable to work with the second Piola-Kirchhoff stress tensor $\boldsymbol{S} . \dot{\boldsymbol{P}}$ and $\dot{\boldsymbol{S}}$ are related to each other through the following relation

$$
\dot{P}_{i j}=\dot{S}_{i r} F_{r j}^{T}+S_{i r} \dot{F}_{r j}^{T}
$$

where $\boldsymbol{F}$ is the deformation gradient tensor and it is defined as

$$
F_{i j}=\frac{\partial x_{i}}{\partial X_{j}}
$$

Substituting Eq.(25) into Eq.(24), and considering an infinitesimal increment of force yields

$$
{ }^{T L} \delta \dot{f}_{I i}^{i n t}=\underbrace{\int_{\Omega_{0}} \frac{\partial N_{I}}{\partial X_{j}} F_{i r} \delta \dot{S}_{j r} d \Omega_{0}}_{T L}+\underbrace{\int_{\Omega_{0}} \frac{\partial N_{I}}{\partial X_{j}} S_{j r} \delta \dot{F}_{i r} d \Omega_{0}}_{T L}
$$

where ${ }^{T L} \delta \dot{\boldsymbol{f}}^{m}$ is the material contribution to the variation of the internal forces and it accounts for the material response through $\dot{\boldsymbol{S}}{ }^{T L} \delta \dot{\boldsymbol{f}}^{g}$ is the geometric contribution and it contains the information of the updated stress field.

\section{Material tangent matrix}

Thanks to the symmetry of $\dot{\boldsymbol{S}}$, the material part ${ }^{T L} \delta \dot{\boldsymbol{f}}^{m}$ of Eq.(27) can be rewritten considering only the symmetric part of the deformation gradient term as follows

$$
{ }^{T L} \delta \dot{\boldsymbol{f}}^{m}=\int_{\Omega^{0}} \boldsymbol{B}_{\mathbf{0}}^{T} \delta \dot{\boldsymbol{S}} d \Omega_{0}
$$


where matrix $\boldsymbol{B}_{0}$ is defined for a node $I$ as

$$
B_{0, I i j k}=\operatorname{sym}_{(i, j)}\left(\frac{\partial N_{I}}{\partial X_{i}} F_{k j}\right)
$$

$\dot{\boldsymbol{S}}$ is related to the deformation rate through the tangent constitutive tensor, as

$$
\dot{S}=\left[C^{0}\right] \dot{E}
$$

where $\boldsymbol{C}^{\mathbf{0}}$ is the fourth-order constitutive tensor referred to the original configuration and $\dot{\boldsymbol{E}}$ is the rate of the Green-Lagrange strain tensor. This can be expressed in terms of the nodal velocities vector $\overline{\boldsymbol{v}}$ as

$$
\dot{\boldsymbol{E}}=\boldsymbol{B}_{\mathbf{0}} \overline{\boldsymbol{v}}
$$

In Eq.(31) and in the following, $(\bar{\cdot})$ denotes a nodal value $\left(e . g . \bar{v}_{J s}\right.$ is the $s$-component of the velocity of node $J)$.

Substituting Eqs.(30,31) into Eq.(28), yields

$$
{ }^{T L} \delta \dot{\boldsymbol{f}}^{\boldsymbol{m}}=\int_{\Omega^{0}} \boldsymbol{B}_{\mathbf{0}}^{T}\left[\boldsymbol{C}^{\mathbf{0}}\right] \boldsymbol{B}_{\mathbf{0}} d \Omega_{0} \delta \overline{\boldsymbol{v}}
$$

In order to obtain the increment of the internal forces, the material time derivative of the internal forces increment is integrated over a time step increment $\Delta t$ as

$$
{ }^{T L} \delta \boldsymbol{f}^{m}={ }^{T L} \delta \dot{\boldsymbol{f}}^{m} \Delta t
$$

From Eq.(33) and Eq.(32), we deduce

$$
{ }^{T L} \delta \boldsymbol{f}^{\boldsymbol{m}}=\int_{\Omega^{0}} \boldsymbol{B}_{\mathbf{0}}^{T} \Delta t\left[\boldsymbol{C}^{\mathbf{0}}\right] \boldsymbol{B}_{\mathbf{0}} d \Omega_{0} \delta \overline{\boldsymbol{v}}
$$

In order to obtain the material tangent matrix in the UL framework, push-forward transformations are applied over Eq.(34), as explained next.

First of all, the new integration domain is the updated configuration $\Omega$ and, for an infinitesimal volume, this is related to $\Omega_{0}$ as follows

$$
d \Omega_{0}=\frac{d \Omega}{J}
$$

Similarly, the constitutive tensor is referred to the spatial configuration by applying the following tranformation

$$
C_{i j k l}^{0}=F_{m i}^{-1} F_{n j}^{-1} F_{o k}^{-1} F_{p l}^{-1} c_{m n o p}^{\nabla \tau} J
$$

where $\boldsymbol{c}^{\nabla \tau}$ is the Truesdell tangent moduli for the rate of the Cauchy stress $\boldsymbol{\sigma} \nabla$. Finally, the material derivatives are replaced by spatial derivatives defining matrix $\boldsymbol{B}$ as follows

$$
B_{I i j k}=\operatorname{sym}_{(i, j)}\left(\frac{\partial N_{I}}{\partial x_{i}} \delta_{k j}\right)
$$

For the node $I$ of a $2 \mathrm{D}$ element, matrix $\boldsymbol{B}$ is

$$
\boldsymbol{B}_{I}=\left[\begin{array}{cc}
\frac{\partial N_{I}}{\partial x} & 0 \\
0 & \frac{\partial N_{I}}{\partial y} \\
\frac{\partial N_{I}}{\partial y} & \frac{\partial N_{I}}{\partial x}
\end{array}\right]
$$


Substituting Eqs.(26,35-37) into (34), yields

$$
\delta \boldsymbol{f}^{\boldsymbol{m}}=\int_{\Omega} \boldsymbol{B}^{T} \Delta t\left[\boldsymbol{c}^{\nabla \boldsymbol{\tau}}\right] \boldsymbol{B} d \Omega \delta \overline{\boldsymbol{v}}
$$
$[4]$

Therefore, the material tangent matrix for the velocity increments in UL framework reads

$$
\boldsymbol{K}^{\boldsymbol{m}}=\int_{\Omega} \boldsymbol{B}^{T} \Delta t\left[\boldsymbol{c}^{\nabla \boldsymbol{\tau}}\right] \boldsymbol{B} d \Omega
$$

The Truesdell tangent moduli tensor $\boldsymbol{c}^{\boldsymbol{\tau}}$ is computed from the Jaumann tensor $\boldsymbol{c}^{\boldsymbol{J}}$ (Eq.(3)) as follows [4]

$$
c^{\nabla \tau}=c^{\nabla J}-c^{*}
$$

where the fourth order tensor $\boldsymbol{c}^{*}$ is defined as

$$
c_{i j k l}^{*}=\frac{1}{2}\left(\delta_{i k} \sigma_{j l}+\delta_{i l} \sigma_{j k}+\delta_{j k} \sigma_{i l}+\delta_{j l} \sigma_{i k}\right)-\delta_{i j} \sigma_{k l}
$$

The incorporation of tensor $\boldsymbol{c}^{*}$ in the material part of the tangent matrix would induce the loss of symmetry of the overall tangent matrix. For this reason, in this work this component has not been included, and the following approximation of the exact material part of the tangent matrix has been considered

$$
\boldsymbol{K}^{\boldsymbol{m}}=\int_{\Omega} \boldsymbol{B}^{T} \Delta t\left[\boldsymbol{c}^{\nabla \boldsymbol{J}}\right] \boldsymbol{B} d \Omega
$$

\section{Geometric tangent matrix}

The geometric tangent matrix for the UL framework is derived using the same procedure as for the material component.

The geometric part of ${ }^{T L} \delta \dot{\boldsymbol{f}}^{\text {int }}$ (Eq.(27)) reads

$$
{ }^{T L} \delta \dot{f}_{I i}^{g}=\int_{\Omega_{0}} \frac{\partial N_{I}}{\partial X_{j}} S_{j r} \delta \dot{F}_{i r} d \Omega_{0}
$$

where the rate of the deformation gradient $\dot{\boldsymbol{F}}$ is defined as

$$
\dot{F}_{i j}=\frac{\partial N_{J}}{\partial X_{j}} \bar{v}_{J i}
$$

Substituting Eq.(45) into Eq.(44), the geometric component of the internal power in the TL description can be written as

$$
{ }^{T L} \delta \dot{f}_{I i}^{g}=\int_{\Omega_{0}} \frac{\partial N_{I}}{\partial X_{j}} S_{j r} \frac{\partial N_{J}}{\partial X_{r}} d \Omega_{0} \delta \bar{v}_{J i}
$$

Integrating Eq.(46) on time for a time step increment $\Delta t$ and adding a dummy unit matrix [4] yields

$$
{ }^{T L} \delta f_{I i}^{g}=\int_{\Omega_{0}} \frac{\partial N_{I}}{\partial X_{j}} \Delta t S_{j r} \frac{\partial N_{J}}{\partial X_{r}} d \Omega_{0} \delta_{i k} \delta \bar{v}_{J k}
$$

In order to recover the UL form, the Piola identity has to be recalled, i.e.

$$
S_{i j}=F_{i k}^{-1} \sigma_{k l} F_{l j}^{-T} J
$$


Applying the chain rule over the material derivatives of Eq.(47), and using Eqs. $(26,35,48)$ yields

$$
\delta f_{I i}^{g}=\int_{\Omega} \frac{\partial N_{I}}{\partial x_{j}} \Delta t \sigma_{j r} \frac{\partial N_{J}}{\partial x_{r}} d \Omega \delta_{i k} \delta \bar{v}_{J k}
$$

The geometric part of the tangent matrix for the velocity increments is obtained from Eq.(49) as

$$
K_{I J i k}^{g}=\int_{\Omega} \frac{\partial N_{I}}{\partial x_{j}} \Delta t \sigma_{j r} \frac{\partial N_{J}}{\partial x_{r}} d \Omega \delta_{i k}
$$

Matrix $\boldsymbol{K}^{g}$ for nodes $I J$ in Voigt notation is

$$
\boldsymbol{K}_{I J}^{g}=\mathbf{1} \int_{\Omega} \boldsymbol{\beta}_{I}^{T} \Delta t \boldsymbol{\sigma} \boldsymbol{\beta}_{J} d \Omega
$$

For $2 \mathrm{D}$ problems $\boldsymbol{\beta}_{I}=\left[\frac{\partial N_{I}}{\partial x}, \frac{\partial N_{I}}{\partial y}\right]^{T}$.

\section{Dynamic component of the tangent matrix}

In this work the implicit Newmark integration rule has been adopted. In particular, the Newmark parameters chosen are $\beta=\frac{1}{4}$ and $\gamma=\frac{1}{2}$ [4]. According to this unconditionally stable scheme, the accelerations $\dot{\boldsymbol{v}}$ and the displacements $\boldsymbol{u}$ are computed, respectively, as

$$
\begin{aligned}
& { }^{n+1} \dot{\boldsymbol{v}}=\frac{2}{\Delta t}\left({ }^{n+1} \boldsymbol{v}-{ }^{n} \boldsymbol{v}\right)-{ }^{n} \dot{\boldsymbol{v}} \\
& { }^{n+1} \boldsymbol{u}={ }^{n} \boldsymbol{u}+\frac{\Delta t}{2}\left({ }^{n+1} \boldsymbol{v}+{ }^{n} \boldsymbol{v}\right)
\end{aligned}
$$

Replacing Eq.(52) into the dynamic term $f_{I i}^{d y n}$ of Eq.(22), and differentiating for the increment of velocities, the dynamic component of the tangent matrix, also known as the mass matrix, is obtained as

$$
K_{I J i j}^{\rho}=\delta_{i j} \int_{\Omega} N_{I} \frac{2 \rho}{\Delta t} N_{J} d \Omega
$$

Or also

$$
\boldsymbol{K}_{I J}^{\rho}=\mathbf{1} \int_{\Omega} N_{I} \frac{2 \rho}{\Delta t} N_{J} d \Omega
$$

\section{V-element}

The solution scheme of the V-element constists on solving iteratively the linear momentum equations for the velocity increments, using the linearization described in the previous section. For each iteration $i$ the following linear system of equations is solved

$$
\boldsymbol{K}^{i} \boldsymbol{\Delta} \overline{\boldsymbol{v}}=\boldsymbol{R}^{i}
$$

with

$$
\boldsymbol{K}^{i}=\boldsymbol{K}^{m}\left({ }^{n+1} \overline{\boldsymbol{x}}^{i}, \boldsymbol{c}^{\nabla J}\right)+\boldsymbol{K}^{g}\left({ }^{n+1} \overline{\boldsymbol{x}}^{i}, \boldsymbol{\sigma}^{i}\right)+\boldsymbol{K}^{\rho}\left({ }^{n+1} \overline{\boldsymbol{x}}^{i}\right)
$$

where $\boldsymbol{K}_{I J}^{m}$ is Eq.(43), $\boldsymbol{K}_{I J}^{g}$ is Eq.(51) and $\boldsymbol{K}_{I J}^{\rho}$ is Eq.(55) and

$$
R_{I k}^{i}=\int_{\Omega} N_{I} \rho N_{J} d \Omega \overline{\dot{v}}_{J k}^{i}+\int_{\Omega} \frac{\partial N_{I}}{\partial x_{j}} \sigma_{k j}^{i} d \Omega-\int_{\Omega} N_{I}^{n+1} b_{k} d \Omega-\int_{\Gamma_{t}} N_{I}^{n+1} t_{k}^{p} d \Gamma
$$

where, for the V-element the Cauchy stress tensor $\boldsymbol{\sigma}$ is computed according to Eq.(16).

Algorithm 1 describes all the steps of the solution scheme for the V-element. 


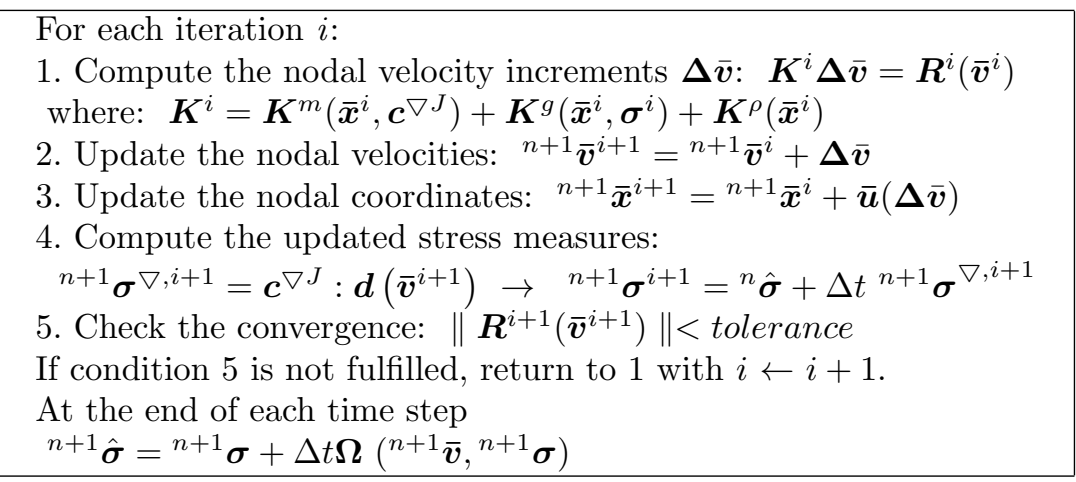

Algorithm 1: Iterative solution scheme for the V-element.

\section{VP-element}

The governing equations of the VP-element are the linear momentum equations and the constitutive relation described by Eq.(15). From the Galerkin approximation of Eq.(15), yields

$$
-\boldsymbol{Q}^{T n+1} \overline{\boldsymbol{v}}+\frac{1}{\Delta t} \boldsymbol{M}_{1}^{n+1} \overline{\boldsymbol{p}}={ }^{n} \boldsymbol{g}
$$

where:

$$
\begin{gathered}
M_{1_{I J}}=\int_{\Omega^{e}} N_{I} \frac{1}{\kappa} N_{J} d \Omega \\
\boldsymbol{Q}_{I J}=\int_{\Omega^{e}} \boldsymbol{B}_{I}^{T} \boldsymbol{m} N_{J} d \Omega \\
{ }^{n} g_{I}=\int_{\Omega^{n}} N_{I} \frac{1}{\kappa \Delta t} N_{J} d \Omega^{n} \bar{p}_{J}
\end{gathered}
$$

where $N$ are linear shape functions, $\boldsymbol{B}$ is the same matrix defined in Eq.(38) and $\boldsymbol{m}=[1,1,1,0,0,0]^{T}$

The whole problem is solved via a two-step Gauss-Seidel partitioned iterative scheme. First the momentum equations are solved in terms of velocity increments. Then Eq.(59) is solved for the nodal pressures using the updated velocities computed with the momentum equations. Concerning the stresses, the Cauchy stress tensor is computed as the sum of its deviatoric part and the pressure using Eq.(17).

In conclusion, for a general time interval $\left[{ }^{n} t,{ }^{n+1} t\right]$ of duration $\Delta t$ the following linear systems are solved for each iteration $i$

$$
\begin{aligned}
\boldsymbol{K}^{i} \boldsymbol{\Delta} \overline{\boldsymbol{v}}^{i+1} & =\boldsymbol{R}^{i}\left(\overline{\boldsymbol{v}}^{i}, \overline{\boldsymbol{p}}^{i}\right) \\
\frac{1}{\Delta t} \boldsymbol{M}_{1} \overline{\boldsymbol{p}}^{i+1} & =\boldsymbol{Q}^{T} \overline{\boldsymbol{v}}^{i+1}+{ }^{n} \boldsymbol{g}
\end{aligned}
$$


where $\boldsymbol{K}$ is the same tangent matrix as for the velocity formulation (Eq.(57)) and the residual vector $\boldsymbol{R}$ is computed as

$$
\begin{aligned}
R_{I i}^{i}=\int_{\Omega} N_{I} \rho N_{J} d \Omega \overline{\dot{v}}_{J i}^{i}+\int_{\Omega} \frac{\partial N_{I}}{\partial x_{j}} \sigma_{i j}\left(\overline{\boldsymbol{v}}^{i}, \overline{\boldsymbol{p}}^{i}\right) d \Omega & -\int_{\Omega} N_{I}^{n+1} b_{i} d \Omega \\
& -\int_{\Gamma_{t}} N_{I}^{n+1} t_{i}^{p} d \Gamma
\end{aligned}
$$

Algorithm 2 describes the iterative solution incremental scheme for the VP-element for a generic time interval $\left[{ }^{n} t,{ }^{n+1} t\right]$.

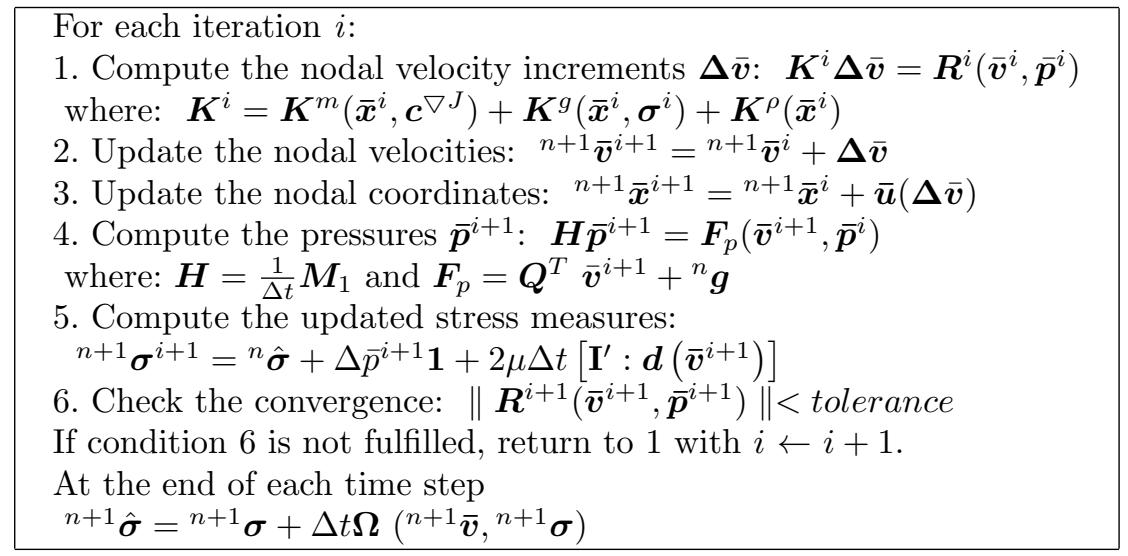

Algorithm 2: Iterative solution scheme for the VP-element.

Note that for the VP-element the material part of the tangent matrix is defined by the same tangent moduli as for the V-element $\left(\boldsymbol{c}^{\nabla J}\right)$. Consequently, the velocity formulation can be easily recovered from the velocity-pressure formulation. In order to do this, the governing equations should be decoupled by computing the Cauchy stress tensor using only the velocities (Eq.(16)) and not as the sum of its deviatoric part and the pressure (Eq.(17)). Note that, if decoupled, the mixed velocity-pressure formulation does not give any advantage with respect to the velocity formulation because the problem is governed by the velocities and the linear momentum equations only.

\section{FIC-stabilization for quasi-incompressible solids}

For incompressible or quasi-incompressible materials, a mixed approach is required in order to properly satisfy the incompressibility constraint. Furthermore in the mixed schemes interpolation functions for the unkown variables must satisfy the inf - sup condition [8], for avoiding numerical instabilities. As previously mentioned, in this work for the mixed VPelement we have used linear shape functions for both the pressure and velocity fields. Thus, for the analysis of quasi-incompressible bodies the scheme has to be stabilized because the inf - sup condition is not fulfilled [8]. 
The VPS-element is stabilized following the FIC approach described in [21] where an analogous Velocity-Pressure scheme for the analysis of quasi-incompressible Newtonian fluids. The analogies between the two formulations are remarkable. First, the schemes are solved for the same governing equations and unknown variables. In fact, one may note that Eq.(64) can represent also the continuity equation for a quasi-incompressible fluid $[27,26,12]$. In particular, for $\kappa=\infty$ the canonical divergence-free continuity equation of the Navier-Stokes problem is recovered. Furthermore, there is a strong analogy between the hypoelastic and the quasi-incompressible Newtonian constitutive laws [17, 13]. For quasi-incompressible Newtonian fluids the stress increment can be computed as $[17,13]$

$$
\Delta \boldsymbol{\sigma}_{f}=2 \mu_{f} \mathbf{I}^{\prime}: \boldsymbol{d}+\Delta t \kappa_{f} \mathbf{1} \otimes \mathbf{1}: \boldsymbol{d}
$$

where $\kappa_{f}$ is the bulk modulus of the fluid and $\mu_{f}$ is the fluid viscosity.

From Eq.(13), the increment of the Cauchy stress for hypoelastic solids is

$$
\Delta \boldsymbol{\sigma}=2 \Delta t \mu \mathbf{I}^{\prime}: \boldsymbol{d}+\Delta t \kappa \mathbf{1} \otimes \mathbf{1}: \boldsymbol{d}
$$

Eqs.(66 and 67 ) show the duality between hypoelastic and Newtonian quasi-incompressible constitutive laws. In Eq.(66) the deviatoric and the volumetric parts of the Cauchy stress tensor are controled by the fluid viscosity $\mu_{f}$ and the bulk modulus $\kappa_{f}$, respectively. The equivalent roles in hypoelastic solids are taken by the second Lamè constant $\mu$ scaled by the time increment and the bulk modulus $\kappa$. Using this equivalence, the same structure of the stabilized mass continuity equation derived for quasi-incompressible fluids in [21] is here used for the analysis of quasi-incompressible hypoelastic solids. This is done by just replacing the fluid parameters $\mu_{f}$ and $\kappa_{f}$ with the equivalent terms $\Delta t \mu$ and $\kappa$ for hypoelastic solids. From this analogy the following stabilized equation for the pressure has been obtained

$$
\left(\frac{1}{\Delta t} \boldsymbol{M}_{1}+\frac{1}{\Delta t^{2}} \boldsymbol{M}_{2}+\boldsymbol{L}+\boldsymbol{M}_{b}\right) \overline{\boldsymbol{p}}=\frac{\boldsymbol{M}_{1}}{\Delta t} \overline{\boldsymbol{p}}+\frac{\boldsymbol{M}_{2}}{\Delta t^{2}}\left({ }^{n} \overline{\boldsymbol{p}}+{ }^{n} \overline{\dot{\boldsymbol{p}}} \Delta t\right)+\boldsymbol{Q}^{T} \overline{\boldsymbol{v}}^{i+1}+\boldsymbol{f}_{p, i}
$$

where the vectors and matrices in Eq.(68) are

$$
\begin{gathered}
M_{2_{I J}}=\int_{\Omega} \tau \frac{\rho}{\kappa} N_{I} N_{J} d \Omega \\
M_{b_{I J}}=\int_{\Gamma_{t}} \frac{2 \tau}{h_{n}} N_{I} N_{J} d \Gamma \\
L_{I J}=\int_{\Omega} \tau\left(\nabla^{T} N_{I}\right) \nabla N_{J} d \Omega \\
f_{p_{I}}=\int_{\Gamma_{t}} \tau N_{I}\left[\rho \dot{v}_{n}-\frac{2}{h_{n}}\left(2 \mu d_{n}-t_{n}\right)\right] d \Gamma-\int_{\Omega^{e}} \tau \nabla^{T} N_{I} \boldsymbol{b} d \Omega
\end{gathered}
$$

where $(\cdot)_{n}$ refer to the normal projection of the variable and the stabilization parameter $\tau$ is given by

$$
\tau=\left(\frac{8 \Delta t \mu}{h^{2}}+\frac{2 \rho}{\delta}\right)^{-1}
$$

where $h$ and $\delta$ are characteristic distances in space and in time, respectively.

The derivation of Eq.(68) is detailed for quasi-incompressible fluids in [21]. 


\section{VPS-element}

The VP and the VPS elements differ only for the pressure equation. For the VP-element the pressure is computed via Eq.(64) while for the VPS-element is computed using the FICstabilized form given in Eq.(68).

The iterative solution incremental scheme for the VPS-element is described for a generic time increment $\left[{ }^{n} t,{ }^{n+1} t\right]$ in Algorithm 3.

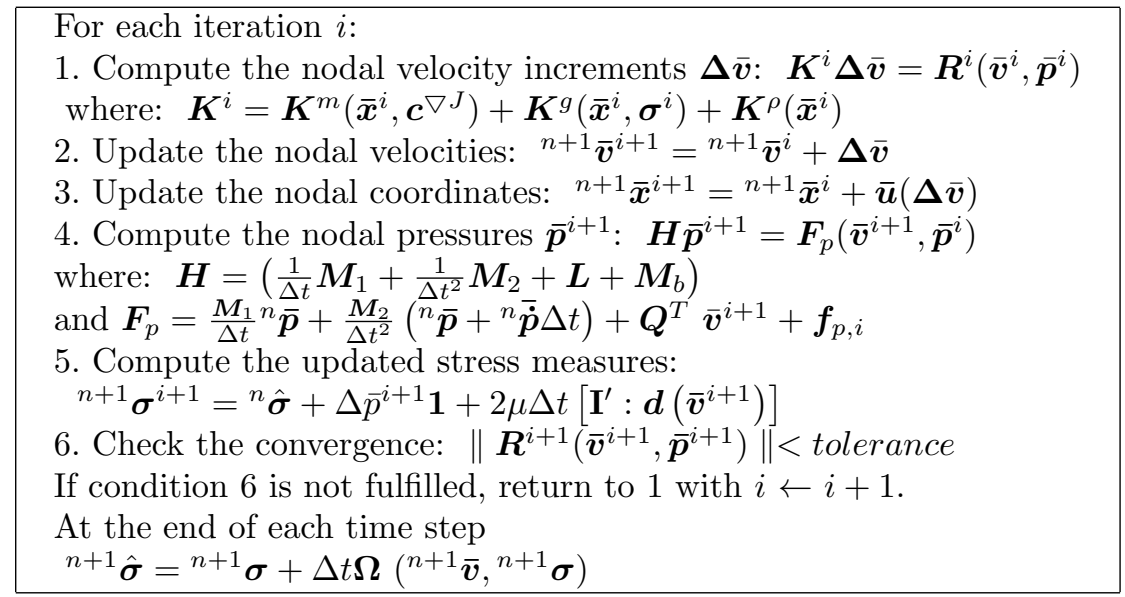

Algorithm 3: Iterative solution scheme for the VPS-element.

\section{Validation examples}

In this section the V, VP and VPS elements are tested against three benchmark problems for non-linear solid mechanics. First, the quasi-incompressible Cook's membrane is analyzed [10]. Then, a uniformly loaded circular plate is solved in statics using the hypoelastic-plastic model described in Section 2. Finally a plane strain cantilever is solved in dynamics for both the hypoelastic and hypoelastic-plastic models. The numerical solutions given by the three solid elements are discussed and compared to the ones published in the literature.

\subsection{Nearly incompressible Cook's membrane}

The Cook's membrane consists of a tapered plate clamped on one of its sides and subjected to a transversal load applied to the opposite side [10]. The membrane is solved considering the Poisson ratio $\nu=0.4999$ and a Young modulus $E=250$. The vertical load is 100 . In Figure 1 the initial geometry and the problem data are given.

The problem was solved in 2D for several unstructured meshes in order to verify the convergence of the scheme. The finest mesh tested in this problem has a mean size of 0.25 and a total of 52372 triangular elements.

The reference solution for this problem is taken from [25], where the problem was solved with 


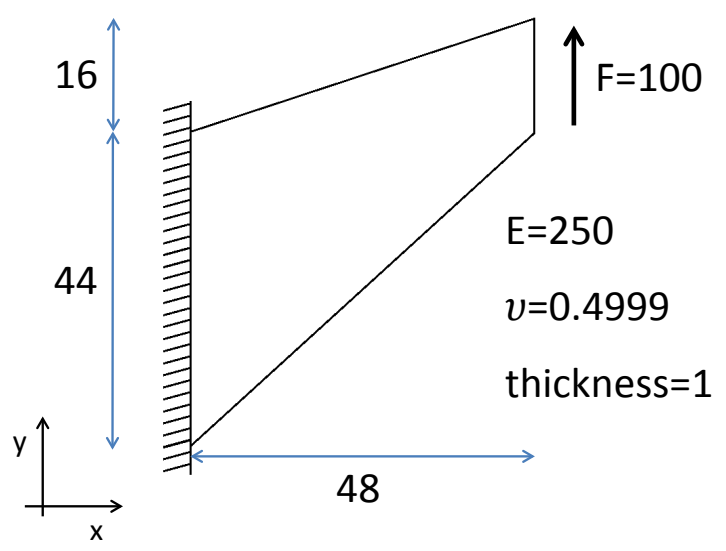

Figure 1: Nearly incompressible Cook's membrane. Initial geometry and problem data.

an Incompatible Bubble element. In the mentioned publication the tip vertical displacement obtained $U_{Y}^{\max }$ by other formulations is also given. Specifically the results are provided for an FEM formulation with linear displacements and constant pressure, and the Enhanced Assumed Strain formulation $[29,5]$. For all these formulations and the finest mesh tested in [25], this value is around $U_{Y}^{\max }=7.71$.

The Poisson ratio 0.4999 represents a material that is almost incompressible. It is well known that values of the Poisson ratio close to 0.5 generate numerical problems to non-stabilized FEM schemes, such as the locking of the solution. Apart from this, the proximity to the incompressible limit also produces ill-conditioned matrices and deteriorates the convergence of the solution. In order to overcome these drawbacks a mixed stabilized formulation is required. For this reason, it is expected that only the VPS-element can give a fine solution to this problem. However the problem is also solved here using the non-stabilized V and VP elements. Both these elements, although in different ways, suffer from instability near the incompressible limit of the material. Despite that, this example is presented with the purpose of showing how each formulation is affected by the mentioned drawbacks and to underline the superiority of a mixed formulation for dealing with nearly incompressible materials.

In Figure 2 the top corner vertical displacements obtained with the V, the VP and the VPS elements for different FEM meshes are plotted.

In Table I the numerical values are given.

From the kinematic point of view, the displacements obtained by the mixed formulation (VP and VPS elements) are close to the expected solution, while the solution given by the V-element locks. For the V, VP and VPS the percentage errors for the tip displacement versus the reference solution are $5.68 \%, 0.707 \%$ and $0.791 \%$, respectively.

For the analysis of stress results, the mesh with average size 1.5 is studied. In Figure 3 the $x x$-component of the Cauchy stress tensor obtained with the V, VP, and VPS elements is plotted over the deformed configurations. The figures show that both V and VP solutions deteriorate for values of $\nu$ close to the incompressible limit of the material, while the stress field given by the VPS-element is good and smooth.

In Figure 4 the pressure solution obtained with the VP-element and the VPS-element are given. 


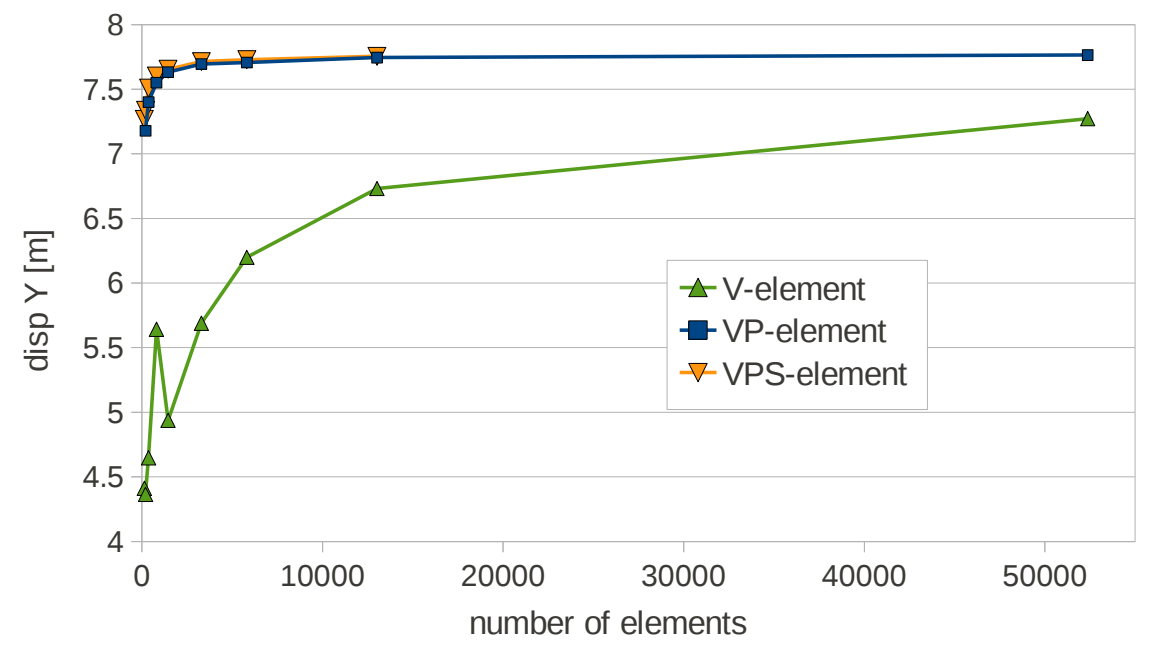

Figure 2: Nearly incompressible Cook's membrane. Top corner vertical displacement for the V, VP and the VPS elements.

\begin{tabular}{|c|c|c|c|c|}
\hline mesh & number of & V-element & VP-element & VPS-element \\
\cline { 3 - 5 } size & elements & $U_{y}$ & $U_{y}$ & $U_{y}$ \\
\hline 5 & 127 & 4.411 & 7.031 & 7.268 \\
4 & 194 & 4.365 & 7.178 & 7.338 \\
3 & 361 & 4.648 & 7.401 & 7.508 \\
2 & 802 & 5.643 & 7.551 & 7.603 \\
1.5 & 1441 & 4.937 & 7.632 & 6.655 \\
1 & 3288 & 5.690 & 7.695 & 7.714 \\
0.75 & 5806 & 6.199 & 7.707 & 7.729 \\
0.5 & 13015 & 6.731 & 7.745 & 7.755 \\
0.25 & 52372 & 7.272 & 7.765 & 7.771 \\
\hline
\end{tabular}

Table I: Nearly incompressible Cook's membrane. Top corner vertical displacement for different formulations and discretizations.

The non-stabilized VP-element yields a pressure field that is completely untrustworthy exhibiting the classical checkerboard modes. Instead, the solution for the VPS element is smooth and accurate. 
(a) V-element

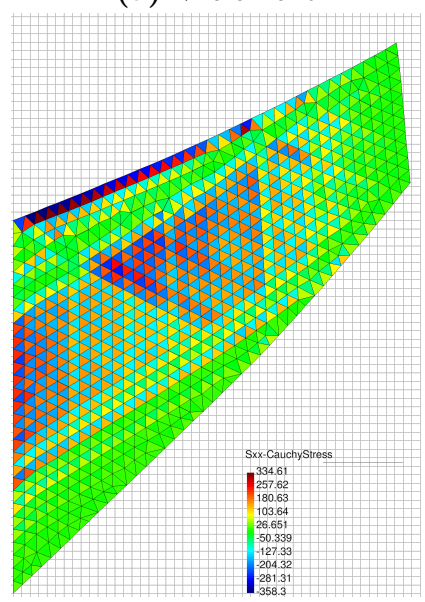

(b) VP-element

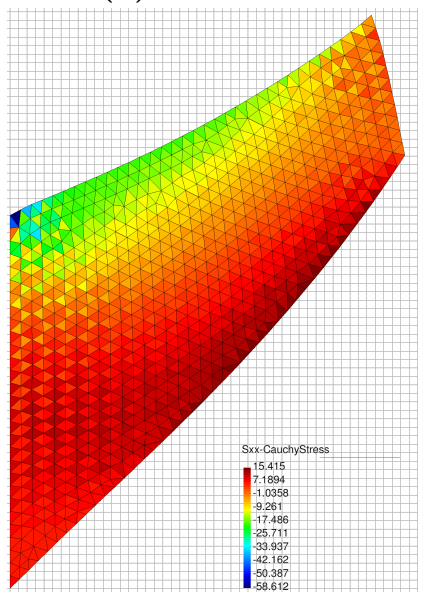

(c) VPS-element

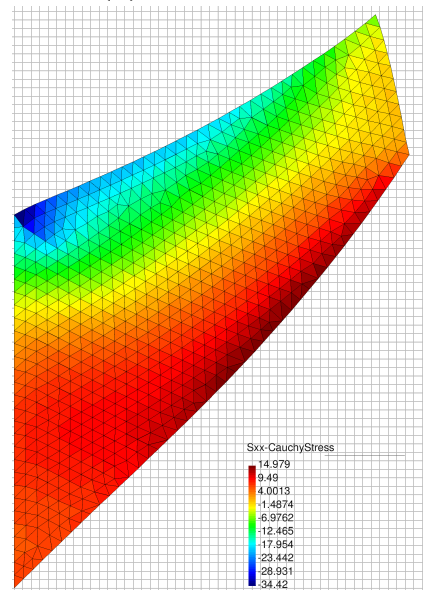

Figure 3: Nearly incompressible Cook's membrane. Results of the XX component of the Cauchy stress tensor for the V, VP and VPS elements.

(a) VP-element

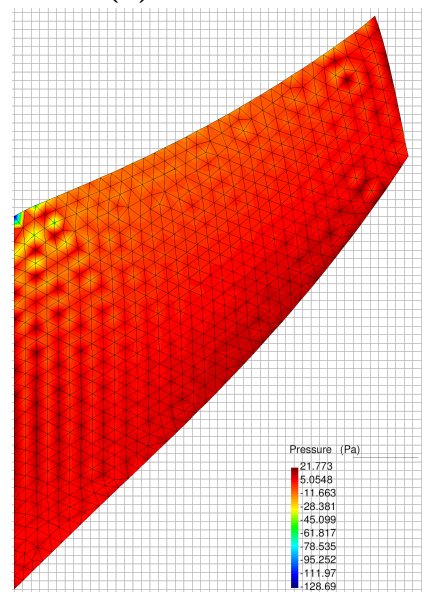

(b) VPS-element

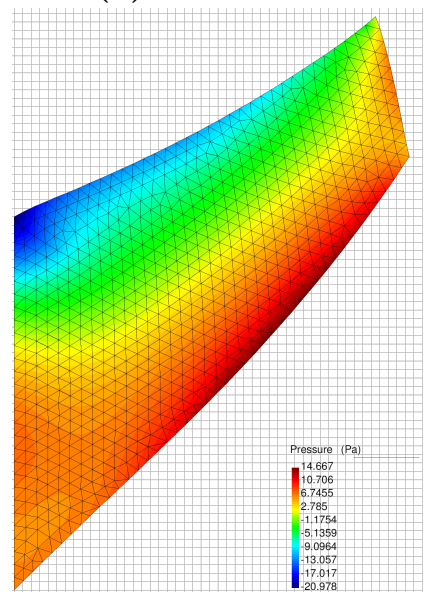

Figure 4: Nearly incompressible Cook's membrane. Pressure solution for the VP and VPS elements. 


\subsection{Uniformly loaded circular plate}

The problem analyzed in this section is a simply supported circular plate subjected to a uniform pressure $P$ on its top surface. The constrains of the plate are applied on its lower edge. The plate has a radius $R=10$ and thickness $h=1$. The problem was presented in [20] and solved exploiting the axisymmetry of the loaded plate. In this work, the axial symmetry of the problem was not used and the plate was analyzed in 3D using 4-noded tetrahedra. The average size for the tetrahedra is 0.175 . This gives 214047 nodes and 1263858 tetrahedra. In Figure 5 the FEM mesh used is shown.

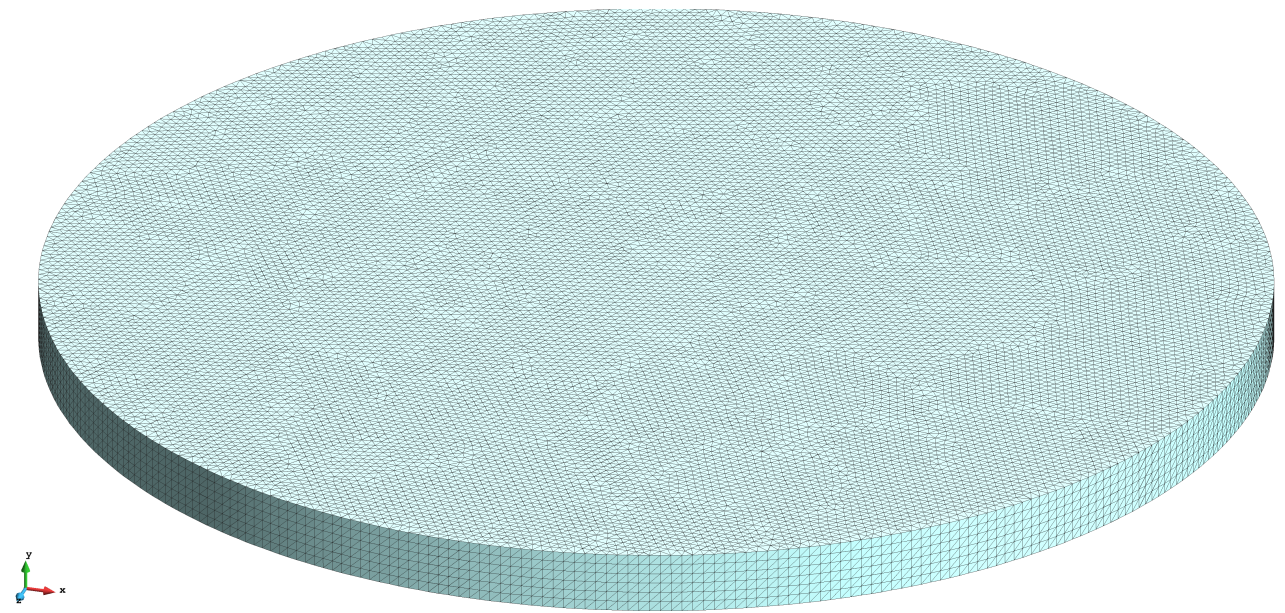

Figure 5: Uniformly loaded circular plate. Initial geometry and 3D FEM mesh used.

A hypoelastic-plastic model with perfect plasticity (plastic modulus $H=0$ ) was used. The problem was solved with the VP-element. For the plastic part a von Mises yield criterion was considered. The plate has Young modulus $E=10^{7}$, Poisson ratio $\nu=0.24$ and uniaxial yield stress $\bar{\sigma}_{y}=16000$. The objective of the study is to determine the limit load for the plate. Using the procedure described in [31], the limit load can be computed analytically by combining the limit analysis and the finite difference method. According to this theory, the limit load can be approximated as

$$
P_{l i m} \approx \frac{1.63 \bar{\sigma}_{y} h^{2}}{R^{2}}=260.8
$$

The same problem was solved in [20] using eight-noded axisymmetric quadrilateral elements with four Gauss integration points. The limit load obtained with a relatively coarse mesh (10 finite elements distributed in two layers across the thickness) is $P_{\text {lim }}^{F E}=259.8$ [20].

As in [20], the limit load has been considered as the one for which the non-linear procedure can not longer converge for a small increment of the load.

In Figure 6 the maximum vertical displacement of the plate is plotted against the pressure on the top surface. In Table II the numerical values are given.

For the present analysis the limit load obtained is $P_{l i m}=264.27$. The relative percentage errors versus the solutions given in [31] and [20] are $1.37 \%$ and $1.76 \%$, respectively. 


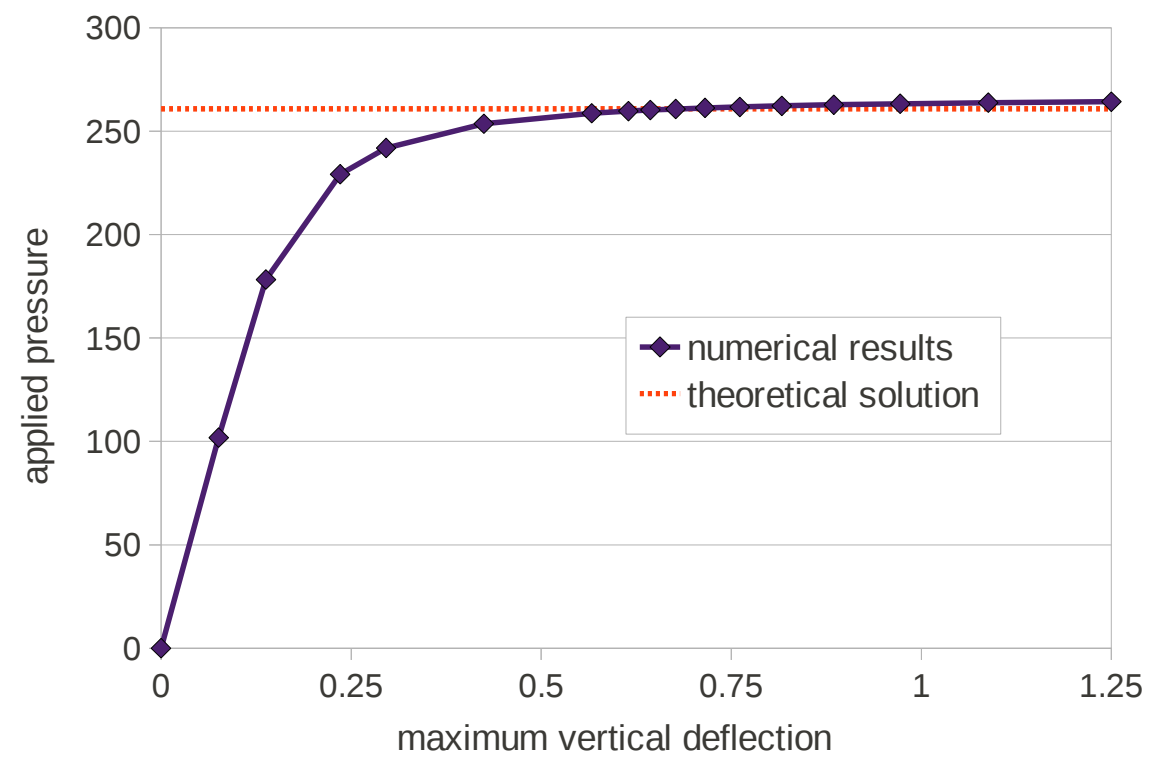

Figure 6: Uniformly loaded circular plate. Maximum deflection versus the applied pressure.

\begin{tabular}{|c|c||c|c|}
\hline pressure & max. deflection & pressure & max. deflection \\
\hline 101.84 & 0.0758 & 260.71 & 0.677 \\
178.22 & 0.138 & 261.21 & 0.716 \\
229.14 & 0.236 & 261.73 & 0.761 \\
241.87 & 0.296 & 262.24 & 0.816 \\
253.58 & 0.424 & 262.73 & 0.885 \\
258.67 & 0.567 & 263.26 & 0.972 \\
259.69 & 0.615 & 263.77 & 1.088 \\
260.20 & 0.644 & 264.27 & 1.250 \\
\hline
\end{tabular}

Table II: Uniformly loaded circular plate. Numerical values of the maximum vertical deflection for different applied pressures.

In Figure 7 the vertical displacements are depicted over the deformed configuration obtained with the limit load. The plate central section is highlighted in the picture.

In Figure 8 some snapshots of the von Mises effective stress are plotted over the central section of the plate for the different load conditions. The picture shows clearly the progressive evolution of the plastic zone. 


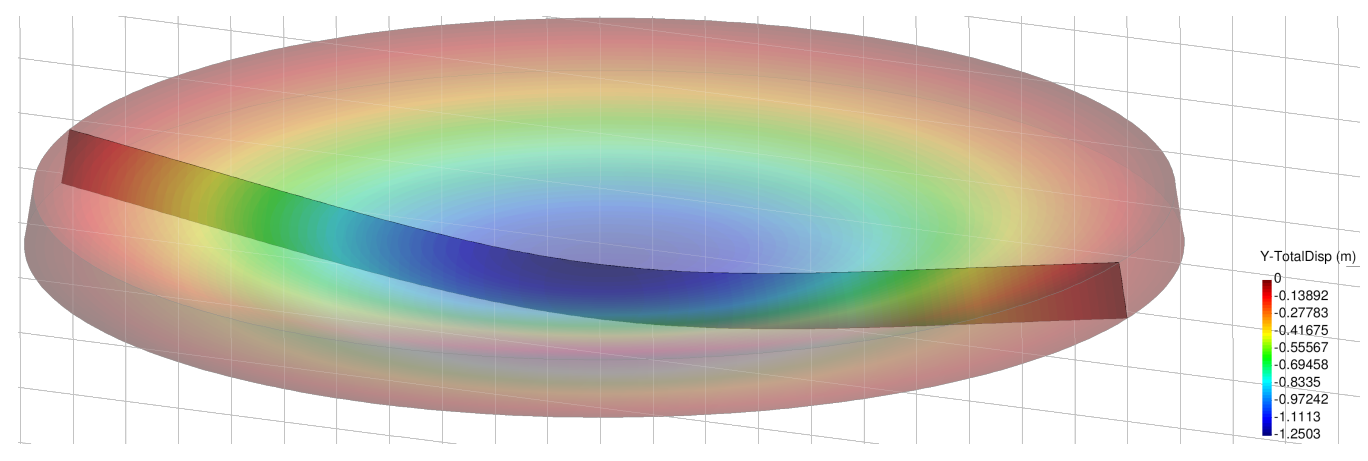

Figure 7: Uniformly loaded circular plate. Vertical displacement contours for the maximum pressure sustained by the plate $\left(P_{l i m}=264.27\right)$. 
(a) Overall load $=101.84$

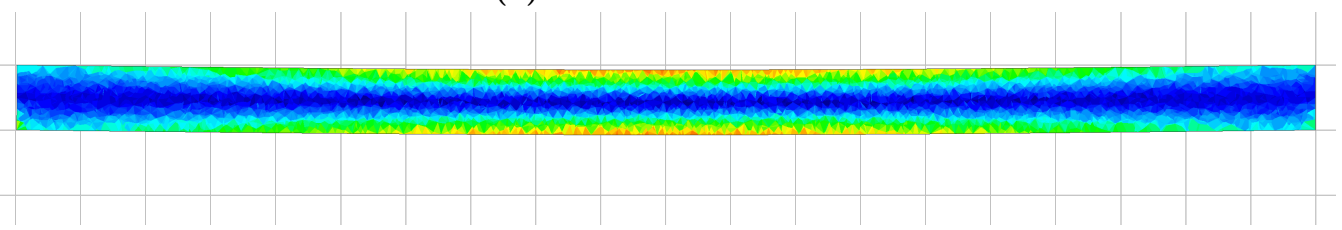

(b) Overall load $=178.22$

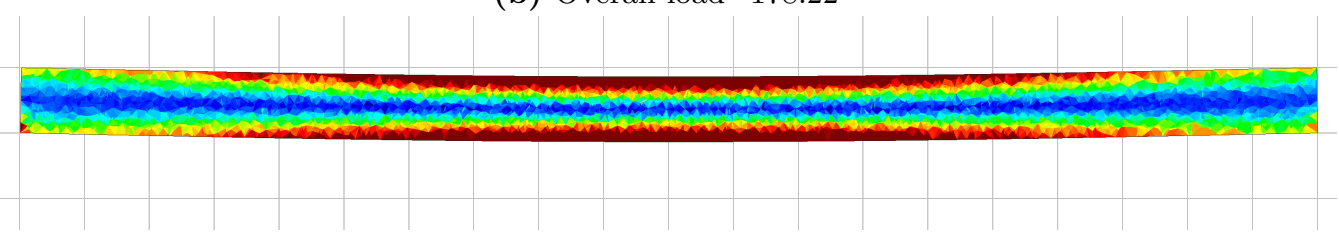

(c) Overall load $=229.14$

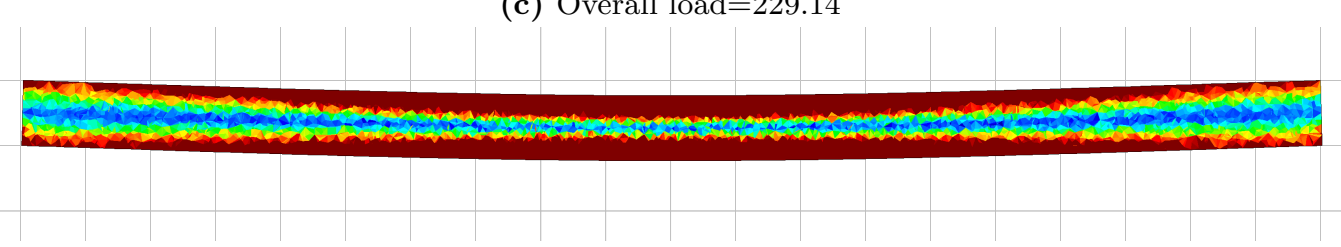

(d) Overall load $=253.58$

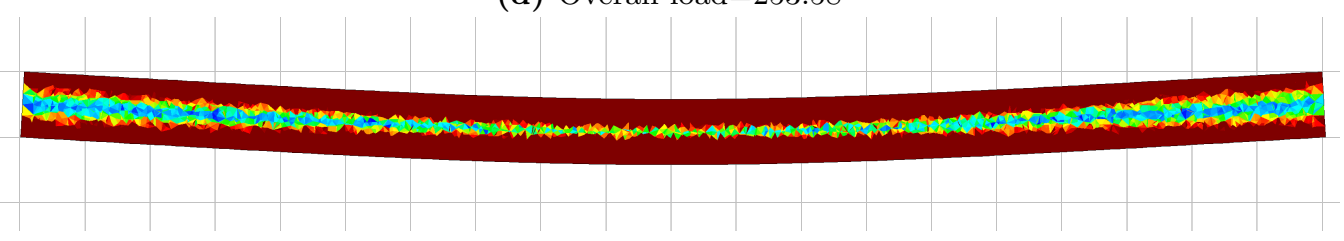

(e) Overall load $=261.73$

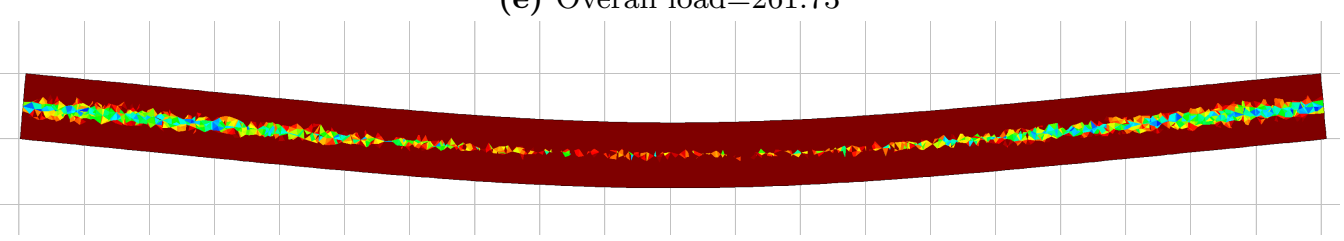

(f) Overall load $=264.27$

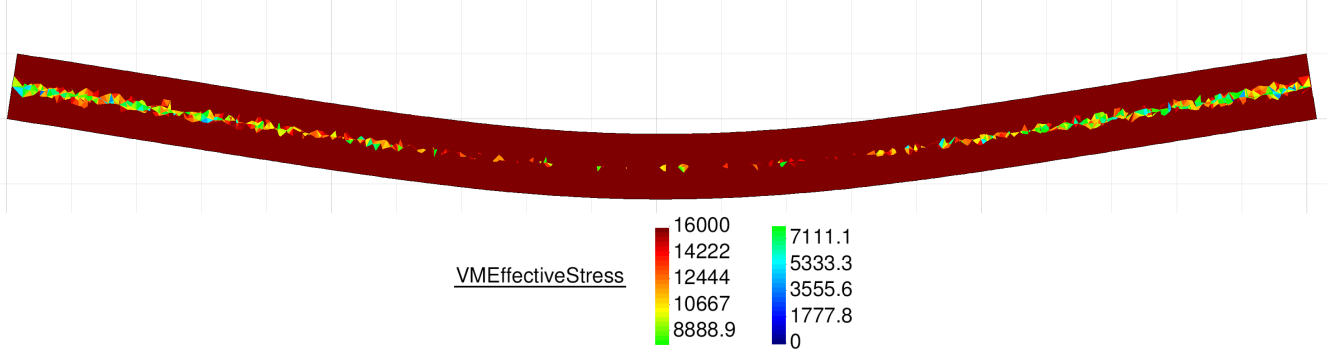

Figure 8: Uniformly loaded circular plate. Von Mises effective stress over the deformed configurations for different load conditions (only the central section is depicted). 


\subsection{Plane strain cantilever in dynamics}

The plane strain cantilever illustrated in Figure 9 was chosen as the reference case for a large displacement dynamics analysis.

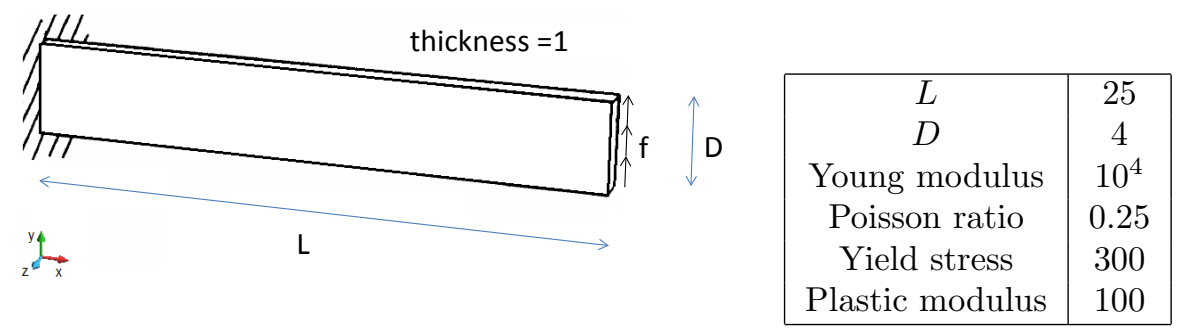

Figure 9: Plane strain cantilever. Initial geometry.

Table III: Plane strain cantilever. Problem data.

The distributed load $f$ is applied on the free edge as a step function at time $t=0$ and its overall value is 40 . The problem data are in given in Table III. The problem was proposed and studied in [3] and here is solved with both a hypoelastic and a hypoelastic-plastic models. First the results of the hypoelastic model are given.

Hypoelastic model

The problem was solved in 2D and 3D and using both the V and VP elements. In order to simulate the plane strain state, in the 3D analysis the nodal displacements in the transversal direction to the load have been constrained [3]. The reference solution is the elastic one given in [3].

The 2D problem was solved for several structured finite element meshes in order to verify the convergence of the scheme.

For the 3D case, the problem was solved only for a structured mesh with average size 0.125 composed by 128004 -noded tetrahedral elements. The results for the 3D case obtained with the VP-element are illustrated in Figure 10. The pressure contours are plotted over the deformed configuration.

In Figure 11 the time evolution of the top corner vertical displacement is plotted for each tested mesh. These results have been obtained with the VP-element.

According to [3], the maximum vertical displacement is $U_{Y}^{\max }=6.88$. This value is very close to the converged results of the V and VP elements. Table IV collects the maximum vertical displacement obtained with the $\mathrm{V}$ and the VP elements for all the meshes.

The four curves plotted in Figure 12 are the converged time evolution of the top corner vertical displacement obtained with the V-element in 3D, the VP-element in 2D and 3D and the reference solution [3]. The curves corresponding to the V and VP elements are almost superposed and they match the reference solution.

Hypoelastic - plastic model

The same problem was solved for an elastic-plastic material with linear hardening. The yield stress is 300 and the plastic modulus $H$ is 100 . The problem was solved with the VP-element and using structured meshes. The reference solution is taken from [3] where the benchmark was proposed. In [3] the converged value for the maximum top corner vertical displacement is 


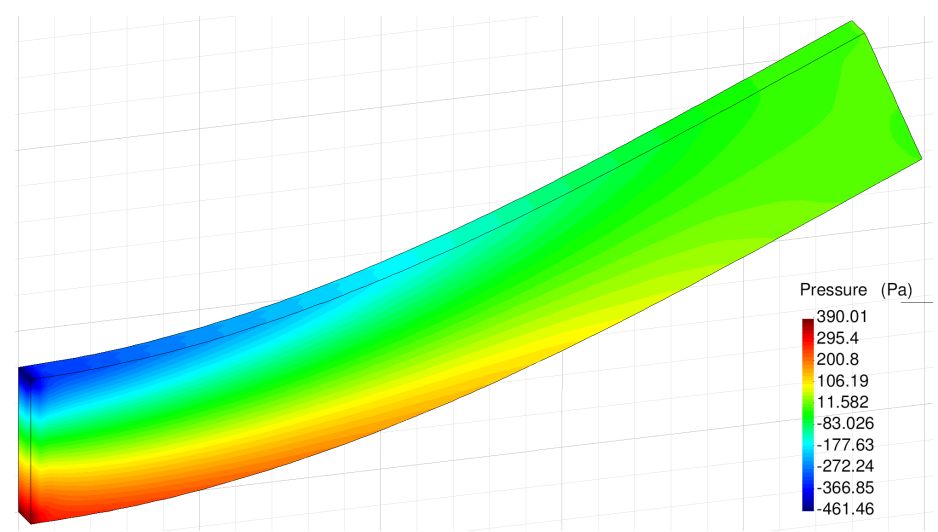

Figure 10: Plane strain cantilever. Numerical results for the $3 \mathrm{D}$ simulation obtained with the VPelement: pressure contours plotted over the deformed configuration.

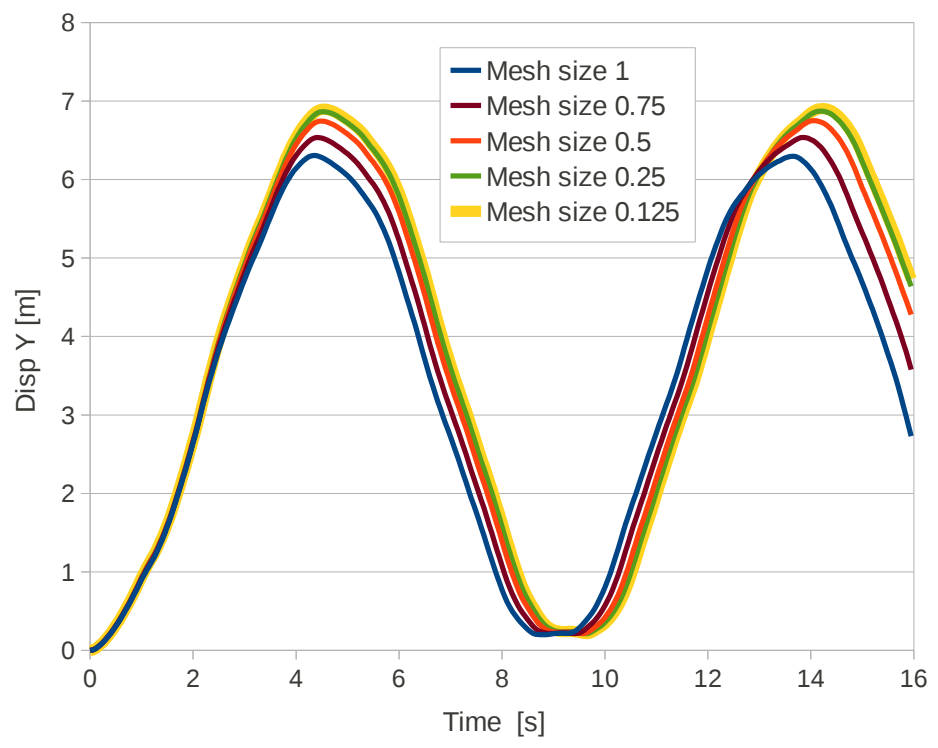

Figure 11: Plane strain cantilever. Time evolution of the top corner vertical displacement for different 2D discretizations. Results obtained with the VP-element.

8.22. The hypoelastic-plastic mixed velocity-pressure formulation converges to 7.97 (error of $2.998 \%$ ). In the graph of Figure 13 the time evolution of the top corner vertical displacement is plotted for the different FEM meshes.

In Table $\mathrm{V}$ the numerical values for the maximum and the residual top corner vertical displacements $\left(U_{y}^{\max }\right.$ and $U_{y}^{r e s}$, respectively) are given for each of the FEM mesh.

The problem was solved also in 3D using a structured mesh of 12800 4-noded tetrahedra with average size 0.125 .

In Figures 14 the von Mises effective stresses are plotted over the deformed configuration at 


\begin{tabular}{|c|c|c|}
\hline mesh & V-element & VP-element \\
\cline { 2 - 3 } size & $U_{y}^{\max }$ & $U_{y}^{\max }$ \\
\hline 1 & 5.759 & 6.306 \\
0.8 & 6.144 & 6.534 \\
0.5 & 6.568 & 6.743 \\
0.25 & 6.811 & 6.863 \\
0.125 & 6.875 & 6.895 \\
\hline
\end{tabular}

Table IV: Plane strain cantilever. Maximum top corner vertical displacement for different 2D discretizations.

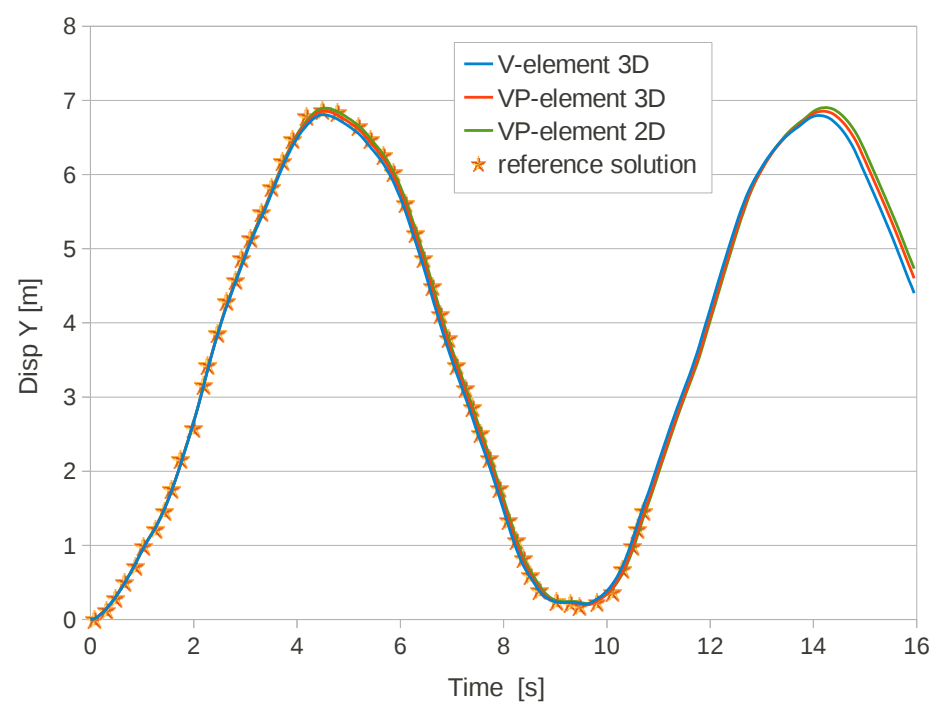

Figure 12: Plane strain cantilever. Time evolution of the top corner vertical displacement. Solutions for the $2 \mathrm{D}$ VP-element and the $3 \mathrm{D} \mathrm{V}$ and VP elements obtained with the finest mesh (average size $0.125)$ compared to the reference solution [3].

\begin{tabular}{|c|c|c|}
\hline mesh size & $U_{y}^{\max }$ & $U_{y}^{\text {res }}$ \\
\hline 1 & 6.77 & 3.25 \\
0.8 & 7.17 & 3.69 \\
0.5 & 7.56 & 4.14 \\
0.25 & 7.82 & 4.51 \\
0.125 & 7.92 & 4.68 \\
0.1 & 7.94 & 4.72 \\
0.0625 & 7.97 & 4.77 \\
\hline
\end{tabular}

Table V: Plane strain elastoplastic cantilever. Maximum and residual top corner vertical displacements for different discretizations. 


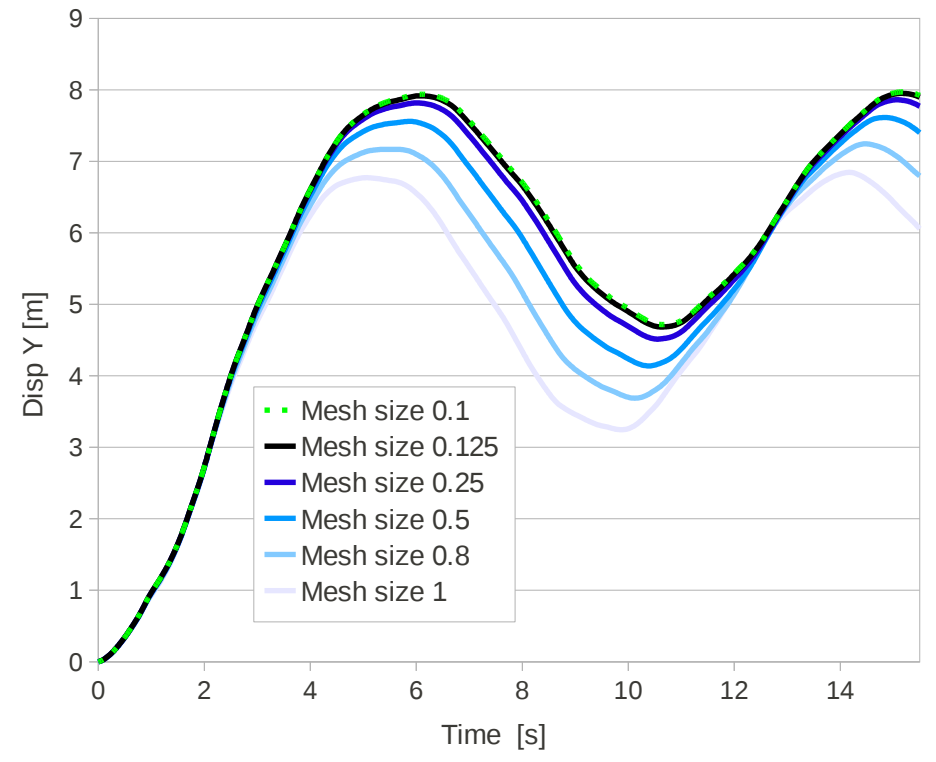

Figure 13: Plane strain elastoplastic cantilever. Time evolution of the top corner vertical displacement for different $2 \mathrm{D}$ discretizations.

the time instant when the maximum top corner vertical displacement is reached $(t=6.05 \mathrm{~s})$.

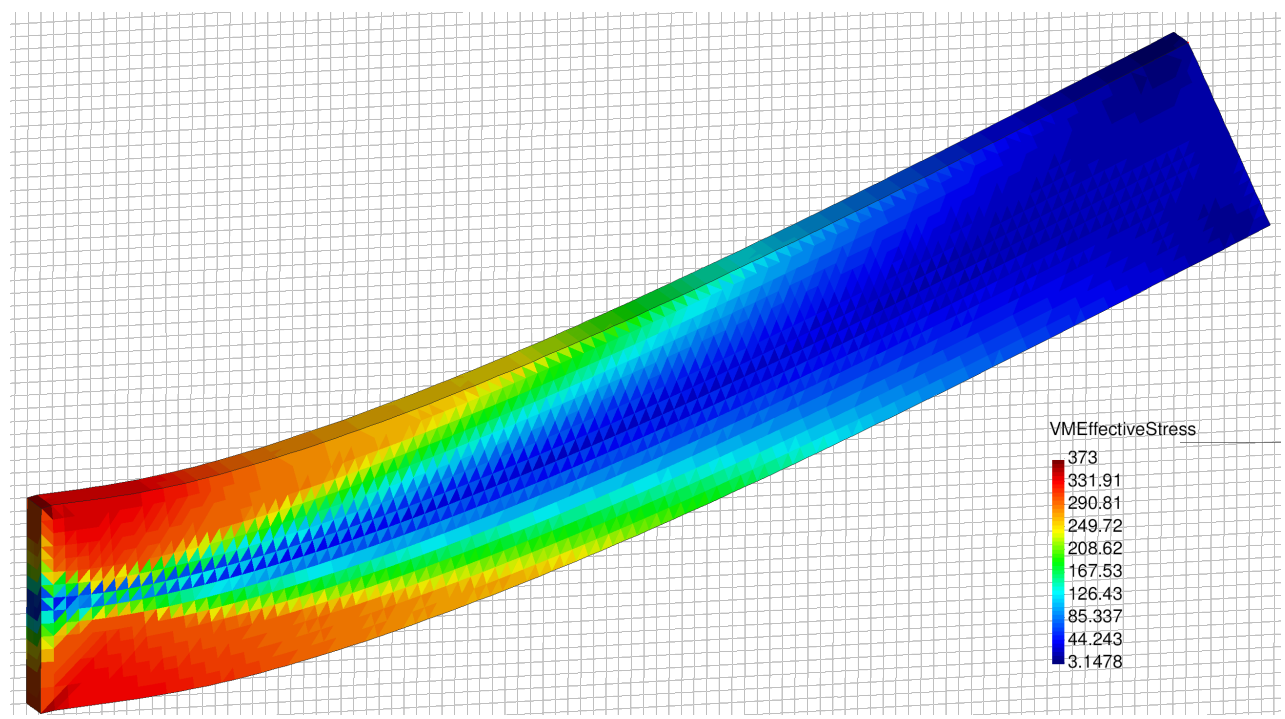

Figure 14: Plane strain elastoplastic cantilever. Numerical results for the 3D simulation. Von Mises effective stress plotted over the deformed configuration at $t=6.05 \mathrm{~s}$.

In Figure 15 for the same time instant the $x x$-component of the Cauchy stress tensor is 
plotted.

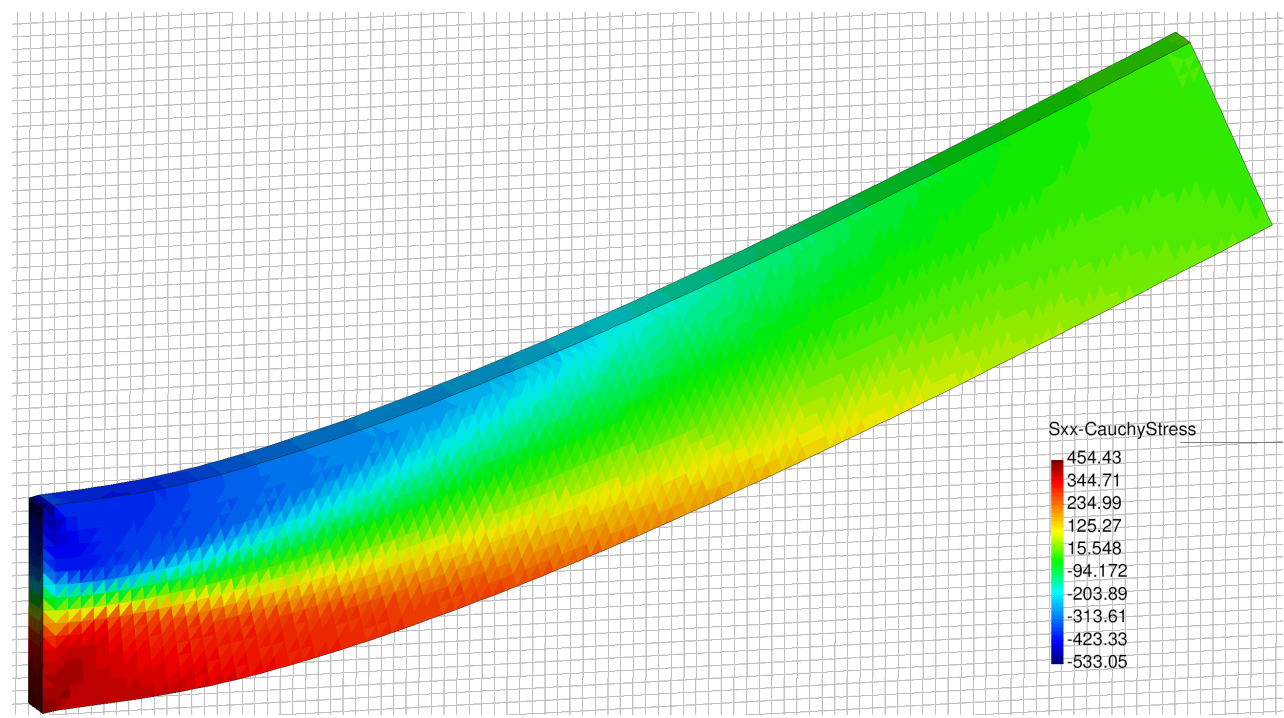

Figure 15: Plane strain elastoplastic cantilever. Numerical results for the 3D simulation. $x x$ component of the Cauchy stress tensor plotted over the deformed configuration at $t=6.05 \mathrm{~s}$.

In Figure 16 the 3D solution is compared to the 2D results obtained with a structured mesh with the same average size. The curves coincide almost exactly.

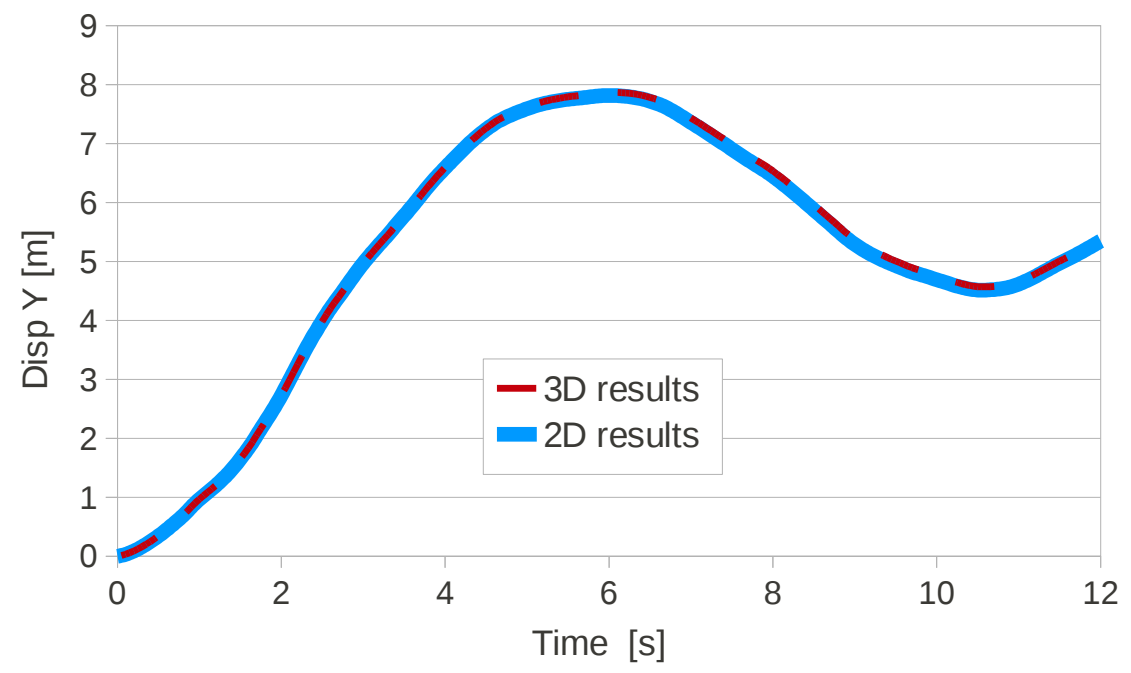

Figure 16: Plane strain elastoplastic cantilever. Time evolution of the top corner vertical displacement. Numerical results for the $2 \mathrm{D}$ and the $3 \mathrm{D}$ simulations for the same average mesh size (0.125). 


\section{Conclusions}

We have presented three velocity-based hypoelastic-plastic finite elements for non-linear solid mechanics. The V-element has been derived from a standard velocity approach, while the VP and VPS elements are based on a mixed velocity-pressure scheme. The VPS-element is stabilized using the Finite Calculus (FIC) method and used for solving problems involving quasi-incompressible materials. The mixed velocity-pressure solution scheme consists on a two-step Gauss-Seidel partitioned iterative algorithm. In particular, first the linear momentum equations are solved in terms of velocity increments, as for the V-element. Then the constitutive relation for the pressure is solved using the updated velocities obtained at the previous step. It has been shown that the proposed hypoelastic elements are capable of solving highly non-linear problems for standard and quasi-incompressible solids with a good accuracy. The numerical results have agreed well with other published results using alternative schemes. The formulation here presented will be very advantageous for solving monolithically FSI problems using the same set of variables for the solid and the fluid [17, 13].

\section{Acknowledgements}

This work was partially supported by the SAFECON Advanced Grant project and the FLOODSAFE Proof of Concept project of the European Research Council. 


\section{REFERENCES}

1. K. J. Bathe. Finite Element Procedures. Prentice-Hall, New Jersey, 1996.

2. P. Becker, S. R. Idelsohn, and E. Oñate. A unified monolithic approach for multi-fluid flows and fluid-structure interaction using the particle finite element method with fixed mesh. Computational Mechanics, 61:1-14, 2015.

3. T. Belytschko and L.P. Bindeman. Assumed strain stabilization of eight node hexahedral element. Computer Methods In Applied Mechanics And Engineering, 105:225-260, 1993.

4. T. Belytschko, W.K. Liu, B. Moran, and K.I. Elkhodadry. Nonlinear Finite Elements For Continua And Structures. Second Edition. John Wiley \& Sons, New York, 2014.

5. M. Bishoff and I. Romero. A generalization of the method of incompatible modes. International Journal for Numerical Methods in Engineering, 69 (9):1851-1868, 2007.

6. J. Bonet, A. J. Gil, and R. Ortigosa. A computational framework for polyconvex large strain elasticity. Computer Methods in Applied Mechanics and Engineering, 283:1061-1094, 2015.

7. T. Brepols, I. N. Vladimirov, and S. Reese. Numerical comparison of isotropic hypo- and hyperelastic-based plasticity models with application to industrial forming processes. International Journal of Plasticity, $63: 18-48,2014$

8. F. Brezzi. On the existence, uniqueness and approximation of saddle-point problems arising from lagrange multipliers. Revue française d'automatique, informatique, recherche opérationnelle. Série rouge. Analyse numérique, 8(R-2):129-151, 1974.

9. M. Cervera, M. Chiumenti, L. Benedetti, and R. Codina. Mixed stabilized finite element methods in nonlinear solid mechanics: Part i: Formulation. Computer Methods In Applied Mechanics And Engineering, 285:752-775, 2015.

10. R. Cook. Improved two dimensional finite element. Journal of the Structural Division, 100:1851-1863, 1974.

11. M. Firat, K. Kaftanoglu, and B.Eser. Sheet metal forming analyses with an emphasis on the springback deformation. Journal of Material Processing Technology, 196 (1-3):135-148, 2008.

12. A. Franci, E. Oñate, and J. M. Carbonell. On the effect of the bulk tangent matrix in partitioned solution schemes for nearly incompressible fluids. International Journal for Numerical Methods in Engineering, 102:257-277, 2015

13. A. Franci, E. Oñate, and J. M. Carbonell. Unified lagrangian formulation for solid and fluid mechanics and fsi problems. Computer Methods in Applied Mechanics and Engineering, 298:520-547, 2016.

14. A. J. Gil, C. H. Lee, J. Bonet, and M. Aguirre. A stabilised petrov-galerkin formulation for linear tetrahedral elements in compressible, nearly incompressible and truly incompressible fast dynamics. Computer Methods In Applied Mechanics And Engineering, 276:659-690, 2014.

15. G Y. Goon, P. I. Poluchin, W. P. Poluchin, and B.A. Prudcowsky. The plastic deformation of metals. Metallurgica, 1968.

16. B. Haddag, T. Balan, and F. Abed-Meraim. Investigation of advanced strain-path dependent material models for sheet metal forming simulations. International Journal of Plasticity, 23 (6):951-979, 2008.

17. S. R. Idelsohn, J. Marti, A. Limache, and E. Oñate. Unified lagrangian formulation for elastic solids and incompressible fluids: Applications to fluid-structure interaction problems via the pfem. Computer Methods In Applied Mechanics And Engineering, 197:1762-1776, 2008.

18. G. Johnson and D.J. Bammann. A discussion of the stress rate in finite deformation problems. International Journal of Solids and Structures, 20:725-737, 1984.

19. J.C. Nagtegaal and J.E. De Jong. Some computational aspects of elastic-plastic large strain analysis. Numerical Methods in Engineering, 17 (1):15-41, 1981.

20. E.A. De Souza Neto, D. Peric, and D.R.J. Owen. Computational methods for plasticity. Theory and applications. John Wiley \& Sons, New York, 2008.

21. E. Oñate, A. Franci, and J. M. Carbonell. Lagrangian formulation for finite element analysis of quasiincompressible fluids with reduced mass losses. International Journal for Numerical Methods in Fluids, 74 (10):699-731, 2014.

22. E. Oñate, A. Franci, and J. M. Carbonell. A particle finite element method for analysis of industrial forming processes. Computational Mechanics, 54:85-107, 2014.

23. E. Oñate, A. Franci, and J. M. Carbonell. A particle finite element method (pfem) for coupled thermal analysis of quasi and fully incompressible flows and fluid-structure interaction problems. Numerical Simulations of Coupled Problems in Engineering. S. R. Idelsohn (Ed.), 33:129-156, 2014.

24. W. Prager. Introduction to Mechanics of Continua. Ginn and Company, Boston, 1961.

25. I. Romero and M. Bishoff. Incompatible bubbles: A non-conforming finite element formulation for linear elasticity. Computer Methods In Applied Mechanics And Engineering, 196:1662-1672, 2006.

26. P. Ryzhakov, J. Cotela, R. Rossi, and E. Oñate. A two-step monolithic method for the efficient simulation of incompressible flows. International Journal for Numerical Methods in Fluids, 74 (12):919-934, 2014. 
27. P. Ryzhakov, R. Rossi, S. R. Idelsohn, and E. Oñate. A monolithic lagrangian approach for fluid-structure interaction problems. Computational Mechanics, 46:883-899, 2010.

28. J. C. Simo and T.J.R. Hughes. Computational Inelasticity. Springer, New York, 1998.

29. J. C. Simo and M. S. Rifai. A class of mixed assumed strain methods and the method of incompatible modes. International Journal for Numerical Methods in Engineering, 29(8):1595-1638, 1990.

30. J. C. Simo and R. L. Taylor. Consistent tangent operators for rate-independent elastoplasticity. Computer Methods in Applied Mechanics and Engineering, 48:101-118, 1985.

31. J. J. Skrzypek. Plasticity and creep. theory, examples, and problems. CRC Press, London, 1993.

32. C. Truesdell. Hypo-elasticity. Journal of Rational Mechanics and Analysis, 4,1, 1955.

33. C. Truesdell. Remarks on hypo-elasticity. Journal of research of the National Bureau of Standards - B. Mathematics and Mathematical Physics, 67B (3):141-143, 1963.

34. O. C. Zienkiewicz and P. N. Godbole. Flow of plastic and visco-plastic solids with special reference to extrusion and forming processes. International Journal for Numerical Methods in Engineering, 8:3-16, 1974.

35. O. C. Zienkiewicz and E. Oñate. Viscoplasticity, plasticity and creep in elastic solids - a unified numerical approach. International Journal for Numerical Methods in Engineering, 8:821-845, 1979. 